\title{
On Demand Responsiveness in Additive Cost Sharing
}

\author{
Hervé Moulin and Yves Sprumont
}

May 24, 2004

\begin{abstract}
A bst ract. We propose two new axioms of demand responsiveness for additive cost sharing with variable demands. G roup M onotonicity requires that if a group of agents increase their demands, not all of them pay less. Solidarity says that if agent $i$ demands more, $j$ should not pay more if $k$ pays less. Both axioms are compatible in the partial responsibility theory postulating Strong Ranking, i.e, the ranking of cost shares should never contradict that of demands. The combination of Strong Ranking, Solidarity and M onotonicity characterizes the quasi-proportional methods, under which cost shares are proportional to 'rescaled' demands.

The alternative full responsibility theory is based on Separability, ruling out cross-subsidization when costs are additively separable. Neither the A umannShapley nor the Shapley-Shubik method is group monotonic. On the other hand, convex combinations of "nearby" ..xed-path methods are group-monotonic: the subsidy-free serial method is the main example. No separable method meets Solidarity, yet restricting the axiom to submodular (or supermodular) cost functions leads to a characterization of the ..xed- $¥$ ow methods, containing the Shapley-Shubik and serial methods.

J EL Classi..cation numbers: C 71, D 63.

Keywords: cost sharing, additivity, cross-subsidization, group monotonicity, solidarity, serial method, Shapley-Shubik method.

Hervé M oulin: Department of Economics, Rice University, M S 22, P.O. Box 1892, Houston Texas 77251-1892, USA; moulin@rice.edu;

Y ves Sprumont: Département de Sciences Economiques, Université de Montréal, C.P. 6128, succursale centre-ville, M ontréal H3C 3J 7, Canada; yves.sprumont@ umontreal.ca

A cknowledgments: We are grateful for their comments to seminar participants at University College London, CORE Louvain-La Neuve, Sabançi and Koç Universities in Istanbul, University of Sydney, University of Melbourne, Universitat A utònoma de Barcelona, and the 2003 Social Choice and Welfare E conomics conference in Malaga. Moulin's research was supported by the NSF under grant SES 011 2032. Sprumont's research was supported by grants from the SSHRC of Canada and the Spanish Ministry of Education.
\end{abstract}


1. Introduction

The traditional model of cooperative games is well suited to discuss cost-sharing problems where the demands of the participating agents are ..xed (inelastic). Shapley (1953) and Weber (1988) showed how the simple axioms of Additivity (with respect to cost) and Dummy (an agent with zero marginal cost pays nothing) determine individual cost shares as a ..xed average of marginal costs.

Following A umann and Shapley's (1974) seminal contribution, the additive theory of cost sharing was extended to variable individual demands: agents $i=1, \ldots, n$ want a quantity $x_{i}$ of good $i$, and the problem is to split the total cost $C\left(x_{1}, \ldots, x_{n}\right)$ using no other information than the cost function $C$. Applications range from the pricing of utilities such as water, phone or electricity, where the level of individual consumption axects total cost, to the sharing of waiting time at a congested server (Shenker (1995), Haviv (2001)), and the division of highway const ruction costs between taxpayers (Lee (2002)).

A simple requirement of demand responsiveness has played a key role in the recent literature on axiomat ic cost sharing. Monotonicity, introduced in Moulin (1995) and developed in Friedman and Moulin (1999), stipulates that every user's cost share should be nondecreasing in her own demand. On the normative side, it expresses a weak form of responsibility for one's own demand. If goods are freely disposable, Monotonicity also has a positive interpretation: it prevents manipulation by arti..cial (and wasteful) increases of individual demands.

In this paper we propose a handful of new demand responsiveness axioms, some of them strengthening Monotonicity, and explore their impact in the additive theory of cost sharing. Our model and our three main axioms are presented in Section 2. We have a ..nite number of users, individual demands are non negative integers, and the cost function is non decreasing, zero for zero demands, and otherwise arbitrary. We always assume that individual cost shares are non negative and depend additively upon the cost function.

Group Monotonicity requires that if a group of agents simultaneously increase their demands, not all of them pay less. Under the same premises, Strong Group Monotonicity states that the total cost share allocated to the group should not decrease. B oth properties have dual normative/ positive interpretations similar to those of $M$ onotonicity. If side-payments are not feasible, Group M onotonicity is suф cient to prevent manipulations by a coordinated arti..cial increase of demands; if they are feasible, we need the latter, stronger property.

Our third main axiom is Solidarity. It requires that a demand change by one agent axects the share of all other agents in the same direction: no one pays more if someone pays less. T he recent literature on fair division explores the Solidarity idea in a number of allocation problems (Thomson (1999), Sprumont and Zhou (1999)), 
but this is its ..rst application to cost sharing.

A natural family of methods meeting all demand resp onsiveness properties above are the quasi-proportional methods computing $i \oint_{S}$ cost share as $\frac{\mathrm{Ps}\left(x_{i}\right)}{s\left(x_{j}\right)} C(x)$, where $s$ is is an arbitrary nondecreasing "scaling function". These simple and familiar methods only take the actual cost into account, and divide it in prop ortion to rescaled individual demands. Theorem 1 in Section 3 characterizes this family by combining Solidarity, Monotonicity, and one more axiom called Strong Ranking. The latter requires cost shares to be ranked in the same order as the corresponding demands, for any cost function, symmetric or not.

Strong Ranking is the de.ning axiom of the "partial responsibility" theory of cost sharing (Moulin and Sprumont (2002)). A gents, while responsible for their demands, are not responsible for the asymmetries of the cost function. This justi..es some cross-subsidization. We submit that this viewpoint inspires cost sharing rules for many publicly provided services: disabled customers are not charged more for transportation services, the same stamp buys delivery of mail to central or remote areas, and so on.

The alternative view that agents are responsible for cost asymmetries is the basis of the more familiar "full responsibility" theory. This view leads to the principle of no cross subsidization pervading the natural monopoly literature (Baumol, Panzar and Willig (1982), Sharkey (1982)), and to the related Dummy axiom in the model of cooperative games. In our setting the relevant formulation is the Separability axiom: if the cost function is additively separable $\left(C(z)={ }_{i} c_{i}\left(z_{i}\right)\right.$ for all $\left.z\right)$, each agent should pay her stand-alone cost $c_{i}\left(x_{i}\right)$.

The impact of our demand responsiveness conditions under full responsibility is the subject of Sections 4 and 5 . P roposition 2 states that neither Sol idarity nor Strong Group M onotonicity is compatible with Separability. On the other hand G roup M onotonicity is a very demanding yet achievable property. The simple method charging incremental costs according to a ..xed ordering of the users is an example. M ore generally, a ..xed-path method charging incremental costs according to a ..xed ordering of the dixerent units of demand, is group-monotonic. However Group Monotonicity is typically not preserved by convex combinations of cost sharing methods. For instancethe Shapley-Shubik method (Shubik (1962)), applying theShapley value to the stand-al one cooperative game at the given demand pro..le, is not group-monotonic. Thus this property yields a power ful critique of both the oldest cost-sharing method in the literature, and of the most popular one, the Aumann-Shapley method (the latter violates even Monotonicity).

P roposition 3 in Section 4 is an important positive result, allowing us to construct "reasonable" group-monotonic methods in the full responsibility approach. Any convex combination of "nearby" ..xed-path methods is group-monotonic as well. The 
most natural method constructed in this fashion is the subsidy-free serial method (Moulin (1995)), adapting serial cost sharing to our discrete model.

If Solidarity is out of reach in the full responsibility theory, a weaker version of this property is feasible when the cost function is submodular. In that case an increase in user $i^{0} s$ demand is a positive externality on other users, and our Submodular Solidarity axiom in Section 5 rules out changes of opposite signs in their cost shares. We introduce the rich family of ..xed- $\ddagger$ ow methods, corresponding to convex combinations of ..xed-path methods, and containing for instance the Shapley-Shubik and subsidy-free serial methods. Theorem 2 characterizes this family by the combination of Separability, M onotonicity, and Submodular Solidarity.

Section 6 compares our results under partial and full responsibility. While the quasi-proportional methods satisfy Solidarity, the cross exects of a demand shift may not have the expected sign. For instance, if $C$ is supermodular and $x_{i}$ increases, agent $j$ 's cost share, $\frac{\operatorname{Ps}\left(x_{i}\right)}{s\left(x_{k}\right)} C(x)$, may go down. We introduce the Positive Externalities and Negative Externalities axioms: if agent $i$ 's demand raises, all agents $j$ other than $i$ bene.t. when costs are submodular, and suxer when costs are supermodular. We show in Proposition 4 that these properties are essentially incompatible with partial responsibility: no cost-sharing method satis. es Strong Ranking and Positive Externalities, and the only method satisfying Additivity, Strong Ranking and Negative Externalities divides costs equally no matter what. By contrast, the axioms are easy to meet under full responsibility: the ..xed-łow methods as well as the AumannShapley method satisfy Positive and Negative Externalities (Proposition 5).

Section 7 takes a second look at the partial responsibility theory. Moulin and Sprumont (2002) introduce W eak Separability, ruling out cross-subsidization when it is not jmplied by Strong Ranking, namely when the cost function takes the form $C(z)={ }_{i} c\left(z_{i}\right)$. While the quasi-proportional methods violate Weak Separability, the axiom is compatible with partial responsibility, i.e., with Strong Ranking. The simplest example is the cross-subsidizing serial method (Sprumont (1998)). This method is also group-monotonic (Proposition 6). Yet Strong Group Monotonicity is incompatible with the combination of Weak Separability and Strong R anking (P roposition 7).

2. The model and the demand responsiveness axioms

Each agent $i 2 N=\mathrm{f} 1, \ldots, n \mathrm{~g}$ demands an integer quantity $x_{i} 2 \mathrm{~N}=\mathrm{f} 0,1, \ldots \mathrm{g}$ of a personalized good. The cost of meeting the demand pro.le $x 2 \mathrm{~N}^{N}$ must be split among the members of $N$. A cost function is a mapping $C: \mathrm{N}^{N} ! \mathrm{R}_{+}$that is nondecreasing and sat is. .es $C(0)=0$; the set of such mappings is denoted $\mathrm{C}$. A (costsharing) method $\varphi$ assigns to eachpproblem $(C, x) 2 \mathrm{Cf} \mathrm{N}^{N}$ a vector of nonnegative cost shares $\varphi(C, x) 2 \mathrm{R}_{+}^{N}$ such that $\varphi_{i}(C, x)=C(x)$. Wecall $\varphi(., x)$ a (cost-sharing) 
method at $x$.

We use the following notation. Vector inequalities are written $\cdot,<, i$. For any

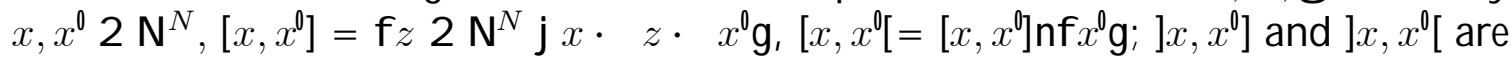
de. ned similarly. We let $N(x)=\mathbf{f} i 2 N \mathbf{j} x_{i}>0 \mathrm{~g}$ and write $n(x)=\mathbf{j} N(x) \mathbf{j} \cdot \mathrm{p}^{\mathrm{F}}$ or any $S \mu N$, we denote by $x_{S} 2 \mathrm{~N}^{S}$ the restriction of $x$ to $S$ and we write $x(S)={ }_{i 2 S} x_{i}$. We de. ne $e^{S} 2 \mathrm{~N}^{N}$ by $e_{i}^{S}=1$ if $i 2 S$ and 0 otherwise. We sometimes write $i$ for fig, $i j$ for $\mathrm{f} i, j \mathrm{~g}$, and i $S$ for $N \mathrm{n} S$. If $i 2 N$ and $C 2$ C, weple..ne $\partial_{i} C: \mathbf{N}^{N} ! \mathrm{R}_{+}$by $\partial_{i} C(z)=C\left(z+e^{i}\right)$ i $C(z)$. Finally, we let $\phi=\mathbf{f} y 2 \mathbf{R}_{+}^{N} \mathbf{j}{ }_{i 2 N} y_{i}=1 \mathrm{~g}$.

The requirement that cost shares be nonnegative is part of our de. nition of a method. Nonnegativity is a minimal form of demand resp onsiveness. It does rule out well-known procedures such as equal split beyond stand-alone costs,

$$
\varphi_{i}(C, x)=C\left(x_{i}, 0_{\mathrm{i}} i\right)+\frac{1}{n}\left(C(x) \mathbf{i}_{j 2 N}^{\mathrm{X}} C\left(x_{j}, 0_{\mathrm{i} j}\right)\right),
$$

and the marginal cost procedure

$$
\varphi_{i}(C, x)=x_{i} \partial_{i} C\left(x \mathbf{i} e^{i}\right)+\frac{1}{n}\left(C(x) \mathbf{i}{ }_{j 2 N}^{\mathbf{X}} x_{j} \partial_{j} C\left(x \mathbf{i} e^{j}\right)\right),
$$

where $\partial_{i} C\left(x \mathbf{i} e^{i}\right)$ is de. ned to be zero if $x_{i}=0$.

Throughout the paper, we restrict our attention to additive methods. Additivity. For any $C, C^{0} 2 \mathrm{C}$ and $x 2 \mathrm{~N}^{N}, \varphi\left(C+C^{0}, x\right)=\varphi(C, x)+\varphi\left(C^{0}, x\right)$.

This powerful mathematical property is devoid of ethical content. A dditive costsharing methods are very convenient in practice. When production can be decomposed into the sum of several independent processes (like research, production and marketing; or construction and maintenance), applying the method to each subprocess and adding the resulting cost shares is equival ent to applying the method to the consolidated cost function. The proper level of application of the method is not a matter of dispute.

We now de. ne four demand resp onsiveness conditions. The ..rst one is the familiar Monot onicity (Moulin (1995)) requiring individual cost shares to be monotonic in the corresponding demands.

Monotonicity. For all $C 2 \mathrm{C}, x, x^{0} 2 \mathrm{~N}^{N}$, and $i 2 N, \mathbf{f} x_{i}<x_{i}^{0}$ and $x_{j}=x_{j}^{0}$ for all $j 2 \mathrm{Nnf} i \mathrm{gg}) \mathrm{f} \varphi_{i}(C, x) \cdot \varphi_{i}\left(C, x^{9} \mathrm{~g}\right.$.

When communication between agents is easy, demand coordination is a concern. We propose a group version of Monotonicity requiring that, when several agents increase simultaneously their demands, not all of them pay less. 
Group M onotonicity. For any $C 2 \mathrm{C}$, any $x, x^{0} 2 \mathrm{~N}^{N}$, and any nonempty $S \mu N$, $\mathbf{f} x_{i}<x_{i}^{0}$ for all $i 2 S$ and $x_{i}=x_{i}^{0}$ for all $\left.i 2 \mathrm{NnSg}\right) \quad \mathbf{f} \varphi_{i}(C, x) \cdot \varphi_{i}(C, x 9$ for at least one $i 2 \mathrm{Sg}$.

A natural strengthening of Group M onotonicity evaluates the impact of demand increases followed by side-payments. When money transfers between agents are feasible, J ohn may be willing to strategically raise his demand if the corresponding drop in some other agents' shares more than compensates the increase in J ohn's share. Strong Group Monotonicity prevents such manipulations.

Strong Group M onotonicity. For any $C 2 \mathrm{C}, x, x^{0} 2 \mathrm{~N}^{N}$, an $p$ nonempty $S \mu$ $\mathrm{P}$ and $i 2 S, \mathrm{f} x_{i}<x_{i}^{0}$ and $x_{j}=x_{j}^{0}$ for all $\left.j 2 N \mathrm{nf} i \mathrm{gg}\right) \mathrm{f}_{j 2 S} \varphi_{j}(C, x)$. ${ }_{j 2 S} \varphi_{j}(C, x 9 \mathrm{~g}$.

Clearly Strong Group Monotonicity also rules out the pro..tability of a coordinated increase by several members of $S$ followed by sidepayments within $S$.

Our last demand responsiveness axiom requires that all members of a group should be treated similarly whenever a demand change occurs outside the group.

Solidarity. For any $C 2 \mathrm{C}$, any $i 2 \mathrm{~N}$, and any $x, x^{0} 2 \mathrm{~N}^{N}, \mathbf{f} x_{j}=x_{j}^{0}$ for all $j 2 \mathrm{Nnf} i \mathrm{gg}) \mathrm{f} \varphi_{j}(C, x) \cdot \varphi_{j}(C, x)$ for all $j 2 \mathrm{Nnf} i \mathrm{~g}$, or $\varphi_{j}(C, x), \varphi_{j}(C, x \mathrm{~g}$ for all $j 2$ Nnf $i$ gg.

We conclude this section by noting the following connection between our axioms. Proposition 1. Every cost-sharing method satisfying Additivity, Solidarity, and M onotonicity satis..es Strong Group Monotonicity.

Proof. The subset $\mathrm{D}=\mathrm{f} C 2 \mathrm{C} \mathrm{j} C(z) 2 \mathrm{f} 0,1 \mathrm{~g}$ for all $z 2 \mathrm{~N}^{N} \mathrm{~g}$ of cost functions taking the values 0 or 1 plays a central role in this and several subsequent proofs, in particular that of Theorem $1^{*}$. E very $C 2 \mathrm{C}$ is a nonnegative linear combination of cost functions in D. Thus an additive method $\varphi$ meets Strong G roup Monotonicity if and only if for all $C 2 \mathrm{D}$ and $x 2 \mathrm{~N}^{N}$,

$$
{ }_{j 2 S} \varphi_{j}(C, x) \cdot{ }_{j 2 S}^{\mathrm{X}} \varphi_{j}\left(C, x+e^{i}\right) \text { for all } S \mu N \text { and all } i 2 S .
$$

If $C(x)=0$, (1) is automatically satis..ed. If $C(x)=1$, then $C\left(x+e^{i}\right)=1$ and (1) is equivalent to

$$
\varphi_{j}\left(C, x+e^{i}\right) \cdot \varphi_{j}(C, x) \text { for all distinct } i, j 2 N,
$$

which is clearly implied by the combination of Solidarity and Monotonicity. 


\section{Demand responsiveness and partial responsibil it $y$ : t he quasi-proportional methods}

This section is devoted to the quasi-proportional methods discussed in the Introduction.

De..nition 1. A method $\varphi$ is quasi-proportional if $\varphi_{i}(C, x)=\frac{\mathrm{p} s\left(x_{i}\right)}{{ }_{2 N} s\left(x_{j}\right)} C(x)$ for all $i, C$, and $x$, where $s: \mathbf{N} ! \mathbf{R}_{+}$is nondecreasing and $s(1)>0$ (with the convention that $\varphi(C, 0)=0)$. Examples include the egalitarian method $\varphi_{i}(C, x)=\frac{1}{n} C(x)$ and the proportional method $\varphi_{i}(C, x)=\frac{x_{i}}{x(N)} C(x)$.

These methods meet Additivity, M onotonicity, and Solidarity, hence, by P roposition 1, satisfy all the demand responsiveness axioms of Section 2. No information about "counter-factual" costs is used in computing the cost shares, which are therefore completely insensitive to the asymmetries in the cost structure. In particular, agents who ask more pay more, regardless of the cost function.

Strong Ranking. For all $C 2 \mathrm{C}, x 2 \mathbf{N}^{N}$, and $\left.i, j 2 N, \mathbf{f} x_{i} \cdot x_{j} \mathbf{g}\right) \quad \mathbf{f} \varphi_{i}(C, x) \cdot$ $\varphi_{j}(C, x) \mathrm{g}$.

This de. nes the "partial responsibility" theory of cost sharing in which agents are held responsible for the size of their own demand but not for the idiosyncrasies of the cost function. Note that the egalitarian method barely satis. es Strong Ranking: it violates the strict version requiring that the ranking of cost shares be identical to that of demands when the cost function is strictly monotonic. The proportional method is more responsive to demands; its simplicity makes it very popular in practice.

We now show that Additivity, Solidarity, M onotonicity and Strong Ranking essentially characterize the quasi-proportional methods. The precise statement requires a couple of additional de..nitions. Notice ..rst that the egalitarian method possesses a property slightly stronger than Solidarity,

Strict Solidarity. For any $C 2 \mathrm{C}$, any $i 2 N$, and any $x, x^{0} 2 \mathbf{N}^{N}, \mathbf{f} x_{j}=x_{j}^{0}$ for all $j 2 N$ nf $i$ gg ) f i) $\varphi_{j}(C, x)<\varphi_{j}\left(C, x 9\right.$ for all $j 2 N$ nf $i \mathrm{~g}$ or ii) $\varphi_{j}(C, x)=\varphi_{j}(C, x 9$ for all $j 2 \mathrm{Nnf} i \mathrm{~g}$ or iii) $\varphi_{j}(C, x)>\varphi_{j}(C, x 9$ for all $j 2 \mathrm{Nnf} i \mathrm{gg}$,

which the prop ortional method fails because agents demanding zero are never axected by changes in others' demands.

On the other hand, the proportional method meets

Zero Cost for Zero Demand. For any $C 2 \mathrm{C}$, any $i 2 N$, and any $x 2 \mathrm{~N}^{N}$, $\left.\mathrm{f} x_{i}=0 \mathrm{~g}\right) \quad \mathrm{f} \varphi_{i}(C, x)=0 \mathrm{~g}$,

which the egal itarian method obviously fails. This axiom is compelling if agents are not responsible for the demands of others ${ }^{1}$.

\footnotetext{
${ }^{1}$ While automatically satis.. ed in the more familiar "full responsibility" theory (where it follows
} 
F inally, we observe that both the egalitarian and the proportional method meet Positive Cost for Positive Demand. For any $C 2 \mathrm{C}$, any $i 2 N$, and any $x 2 \mathrm{~N}^{N}$, $\mathbf{f} x_{i}>0$ and $\left.C(x)>0 \mathbf{g}\right) \quad \mathbf{f} \varphi_{i}(C, x)>0 \mathbf{g}$,

which is a strong but simple interpretation of responsibility for one's demand ${ }^{2}$. We are now ready to state our ..rst main result.

Theorem 1.

i) Assume $n$, 4. A cost-sharing method $\varphi$ satis..es Additivity, Strong Ranking, Strict Solidarity, and $M$ onotonicity if and only if there exists a nondecreasing function $s: \mathrm{N}$ ! R such that $s(0)>0$ and

$$
\varphi_{i}(C, x)=\frac{p s\left(x_{i}\right)}{j 2 N s\left(x_{j}\right)} C(x)
$$

for all $C 2 \mathrm{C}, i 2 \mathrm{~N}$, and $x 2 \mathrm{~N}^{N}$.

ii) A ssume $n$, 3. A cost-sharing method $\varphi$ satis..es Additivity, Strong Ranking, Solidarity, Monotonicity, Zero Cost for Zero Demand and Positive Cost for Positive Demand if and only if there exists a nondecreasing function $s: \mathrm{N}$ ! R such that $s(0)=0, s(1)>0$, and (2) holds for all $C 2 \mathrm{C}, i 2 N$, and $x 2 \mathrm{~N}^{N}$ (with the convention $\varphi(C, 0)=0)$.

Choosing a positive constant $a$ and setting $s(t)=t+a$ for all $t$, equation (2) de. nes a hybrid method approaching the egalitarian method when $a ! 1$ and the proportional one when $a$ ! 0 . A ny such method meets Strict Solidarity.

Four observations are in order.

(1) Theorem 1 is a corollary to Theorem $1^{*}$ and Lemma 2 , stated and proved in the Appendix, which dispense with Strong R anking. Dropping Strong Ranking allows for a family including more complicated methods, of which two interesting subclasses are easily described.

Consider ...rst the methods $\varphi(C, x)=\theta(x) C(x)$, where $\theta(x)$ is an arbitrary mapping from $\mathrm{N}^{N}$ nf $0 \mathrm{~g}$ to $\$$. We call such methods simple: they completely ignore the shape of the cost function and reduce the cost-sharing problem to a "general claim arbitration" problem (where the amount to be divided may exceed or fall short of the sum of the claims) as in Herrero, Maschler and Villar (1999) and Naumova (2002).

from Additivity and Separability: see Section 4), Zero Cost for Zero Demand must be explicitly required in the partial responsibility theory.

${ }^{2}$ This axiom is incompatible with the main axiom of the full responsibility theory, Separability, because a dummy agent pays nothing even if his demand is positive. By contrast, Positive Cost for Positive Demand is easily met in the partial responsibility approach. 
It follows from the proof of Theorem $1^{*}$ that a simple method meets Additivity, Solidarity and Monot onicity if and only if it is an asymmetric quasi-proportional method, i.e.,

$$
\theta_{i}(x)=\frac{\mathbf{p} s_{i}\left(x_{i}\right)}{j 2 N s_{j}\left(x_{j}\right)},
$$

for all $i 2 N$, where $s_{i}: \mathbf{N} ! \mathbf{R}_{+}$is nondecreasing and $s_{i}(1)>0$.

A second interesting subclass meeting our three axioms consists of the methods

$$
\varphi_{i}(C, x)=\frac{\lambda}{n \mathbf{i} 1} C\left(x_{i}, 0_{\mathbf{i}}\right)+\frac{1}{n}\left(C(x) \mathbf{i}{ }_{j 2 N}^{\mathbf{X}} \frac{\lambda}{n \mathbf{i} 1} C\left(x_{j}, 0_{\mathbf{i} j}\right)\right)
$$

for all $i, C$, and $x$, where $\lambda 2[0,1]$. For $\lambda=0$, this is just the egalitarian method. Setting $\lambda=n \mathbf{i} 1$ yields "equal split beyond stand-al one costs" introduced in Section 2 , which however does not guarantee nonnegative cost shares. The nonnegativity constraints force $\lambda$ between 0 and 1 . These methods split equally the bal ance above a fraction of stand-al one costs. The entire family of methods meeting A dditivity, M onotonicity and Solidarity combines feat ures of these methods and the quasi-proportional methods: see the A ppendix for details.

(2) Replacing the combination of Solidarity and M onotonicity by Strong Group Monot onicity (which is implied: recall Proposition 1) allows for many more methods. For instance, the simple methods $\varphi(C, x)=\theta(x) C(x)$ satisfy Strong Group M onotonicity if and only if $\theta_{i}$ is non-increasing in $x_{j}$ for any two distinct $i, j$ (which is much less restrictive than the asymmetric quasi-proportional form (3)) and Strong Ranking if and only if $\theta_{i}(x) \cdot \theta_{j}(x), x_{i} \cdot x_{j}$ for any $i, j$ and $x$. Characterizing the entire class of methods meeting Additivity, Strong Group Monotonicity and Strong Ranking is a challenging open problem.

(3) All methods in Theorem 1 satisfy a requirement stronger than Solidarity. When an arbitrary subset of agents change their demands, the impact on the cost shares of the others goes in the same direction: for any $C 2 \mathrm{C}$, any $S \mu \mathrm{N}$, and any $x, x^{0} 2 \mathbf{N}^{N}, \mathbf{f} x_{j}=x_{j}^{0}$ for all $\left.j 2 \mathrm{NnSg}\right) \quad \mathbf{f} \varphi_{j}(C, x) \cdot \varphi_{j}(C, x q$ for all $j 2 \mathrm{Nn} S$ or $\varphi_{j}(C, x), \varphi_{j}(C, x)$ for all $j 2 \mathrm{NnSg}$.

(4) The egalitarian method occupies a special place within the quasi-proportional methods, because it meets a much stronger version of the Solidarity axiom:

Full Solidarity. For any $C 2 \mathrm{C}$, any $i 2 N$, and any $x, x^{0} 2 \mathbf{N}^{N}, \mathbf{f} x_{i}<x_{i}^{0}$ and $x_{j}=x_{j}^{0}$ for all $\left.j 2 \mathrm{Nnf} i \mathrm{gg}\right) \quad \mathbf{f} \varphi(C, x) \cdot \varphi(C, x \mathbf{g}$.

This axiom strengthens both Solidarity and Monotonicity. It characterizes the ..xed-proportions methods: a cost-sharing method $\varphi$ satis.. es Additivity and Full Sol- 
idarity if and only if there exists $\lambda 2 \&$ such that $\varphi(C, x)=C(x) \lambda$ for all $C 2 \mathrm{C}$ and $x 2 \mathrm{~N}^{N}$. We omit the easy proof ${ }^{3}$.

4. Demand Responsiveness and ful I responsibil it $y$

We turn to demand responsiveness properties within the familiar "full responsibility" approach, where agents are responsible for the idiosyncrasies of the cost function. The de..ning axiom says that everyone should pay the cost of their own demand whenever that cost does not depend on others agents' demands.

Separability. For all $C 2 \mathrm{C}, x 2 \mathrm{~N}^{N}, \mathrm{f} C(z)={ }^{\mathrm{P}}{ }_{i 2 N} c_{i}\left(z_{i}\right)$ for all $z 2 \mathrm{~N}^{N}$, where $c_{i}(0)=0$ for all $\left.i \mathrm{~g}\right) \quad \mathbf{f} \varphi_{i}(C, x)=c_{i}\left(x_{i}\right)$ for all $i 2 \mathrm{Ng}$.

All quasi-proportional methods violate Separability. More fundamentally, the axiom is incompatible with Strong Ranking even in the absence of A dditivity. Suppose costs are additively separable $\left(C(z)=\quad c_{i}\left(x_{i}\right)\right)$ and $i$ 's stand-alone cost exceeds $j$ 's at all levels $\left(c_{i}(t)>c_{j}(t)\right.$ for all $\left.t>0\right)$. If $x_{i}=x_{j}$, Strong $\mathrm{R}$ anking imposes equal cost shares for $i$ and $j$ while Separability requires that $i$ pays more than $j$.

Under Additivity, Separability is equival ent to the more familiar Dummy axiom requiring that dummy agents pay nothing: for all $C 2 \mathrm{C}, x 2 \mathrm{~N}^{N}$, and $i 2 N$, $\left.\mathrm{f} \partial_{i} C=0 \mathrm{~g}\right) \quad \mathrm{f} \varphi_{i}(C, x)=0 \mathbf{g}^{4}$.

The combination of Additivity and Separability is well understood. Moulin and Vohra (2003) oxer a representation in terms of ‡ows. A conservative unit $\ddagger$ ow - or simply, a $\neq$ ow- to a demand pro..le $x \mathbf{2} \mathbf{N}^{N}$ is a mapping $f(., x):\left[0, x\left[!_{\mathrm{P}} \mathbf{R}_{+}^{N}\right.\right.$ satisfying the convention that $f_{i}(z, x)=0$ whepever $z_{i}=x_{i}$, the normalization ${ }_{i 2 N} f_{i}(0, x)=$ 1 , and the conservation constraints ${ }_{i 2 N} f_{i}(z, x)={ }_{i 2 N(z)} f_{i}\left(z \mathbf{i} e^{i}, x\right)$ for all $z 2$ ]0, $x$ [. Note that this implies ${ }_{i 2 N(x)} f_{i}\left(x \mathbf{i} e^{i}, x\right)=1$.

Lemma 1 (Moulin and Vohra (2003)). A method $\varphi$ satis..es Additivity and Separability if and only if, for every $x 2 N^{N}$, there is a (necessarily unique) ‡ow $f(., x)$ to $x$ such that

$$
\varphi_{i}(C, x)={ }_{z 2[0, x[}^{\mathrm{X}} f_{i}(z, x) \partial_{i} C(z)
$$

for all $C 2 C$ and all $i 2 N$; we call $f$ the $\neq$ ow representation of $\varphi$.

A $n$ important consequence of this representation is the following property. Independence of Irrelevant Costs. For all $x 2 \mathrm{~N}^{N}$ and $C^{1}, C^{2} 2 C, \mathrm{f} C^{1}(z)=$ $C^{2}(z)$ for all $\left.z 2[0, x] \mathrm{g}\right) \quad \mathbf{f} \varphi\left(C^{1}, x\right)=\varphi\left(C^{2}, x\right) \mathrm{g}$.

\footnotetext{
${ }^{3} \mathrm{~N}$ ote that if $D 2 \mathrm{D}$ and $D(x)=1$, Full Solidarity implies $\varphi(D, x)=\varphi\left(D, x+e^{i}\right)$ for all $i$. This implies ... rst that $\varphi\left(\delta^{x}, x\right)$ is independent of $x$, then $\varphi(D, x)$ is independent of both $D$ and $x$.

${ }^{4}$ See M oulin and Vohra (2003), Corollary 3, and notice that under A dditivity, their Non-Dummy axiom is equivalent to Separability.
} 
The simplest separable additive methods correspond to $\ddagger$ ows $f(., x)$ mapping $[0, x$ [ into f $0,1 \mathrm{~g}^{\mathrm{N}}$. In this case the entire fow runs along a monotone path from 0 to $x$ in the integer grid. Fix such a path. At each step of the path, the coordinate of exactly one agent increases by one. Charging to this agent the corresponding cost increase and summing over the entire path de.nes a cost-sharing method at the demand pro.l.e $x$. Formally, any sequence of agents $\pi: \mathrm{f} 1, \ldots, x(N) \mathrm{g} ! N$ such that $\mathbf{j} \pi^{\mathrm{i}}{ }^{1}(i) \mathbf{j}=x_{i}$ for each $i 2 \mathrm{~N}$, de. nes a monotone path $z^{\pi}$ to $x$ by setting $z^{\pi}(0)=0$ and $z^{\pi}(t)=z^{\pi}(t ; 1)+e^{\pi(t)}$ for $t=1, \ldots, x(N)$. This path, in turn, generates the fow $f_{i}(z, x)=1$ if for some $t, z^{\pi}(t ; \mathbf{i} 1)=z, z^{\pi}(t)=z+e^{i}$, and $f_{i}(z, x)=0$ otherwise. The corresponding cost shares at $x$ are

$$
\varphi_{i}^{\pi}(C, x)=\partial_{t 2 \pi^{i}{ }^{1}(i)}^{\mathrm{X}} \partial_{i} C\left(z^{\pi}(t ; \quad 1)\right)
$$

for all $i 2 N$ and $C 2 \mathrm{C}$. If, for every demand pro..le $x, \varphi(., x)$ is generated by a monotone path to $x$, we say that $\varphi$ is path-generated. Wang (1999) shows that a method satis..es Additivity and Separability if and only if it is the pointwise limit of convex combinations of path-generated methods. Note that if we restrict attention to a bounded subset of demand pro..les, the two axioms characterize simply the (..xed) convex combinations of path-generated methods.

Example 1. The Aumann-Shapley ${ }^{5}$ method $\varphi^{a s}$ solves every problem $(C, x)$ by averaging the cost-share vectors $\varphi^{\pi}(C, x)$ generated along all paths $\pi$ to $x$. In the corresponding łow representation, $f_{i}^{a s}(z, x)$ equals the proportion of paths to $x$ which go through $z$ and $z+e^{i}$ : straightforward computation $\mathcal{Q}$ yield $f_{i}^{a s}(z, x)=\frac{\alpha(z) \alpha\left(x_{\mathrm{i}} z_{\mathrm{i}} e^{i}\right)}{\alpha(x)}$ for all $i 2 N$ and $z 2\left[0, x \mathbf{i} e^{i}\right.$, where $\alpha(z)=z(N) ! /{ }_{j 2 N} z_{j} !$.

Example 2. The Shapley-Shubik method $\varphi^{s s}$ (Shubik (1962)) averages the cost shares computed along the paths that follow the edges of the cube [0, $x$ [. Letting $E_{i}(x)=\mathbf{f} z 2\left[0, x\left[\mathbf{j} 8 j 2 \mathrm{Nn} i, z_{j} 2 \mathrm{f} 0, x_{j} \mathrm{gg}\right.\right.$, the $\neq$ ow associated with $\varphi^{s s}(., x)$ is given by $f_{i}^{s s}(z, x)=\frac{\left(n(x) \mathrm{i} n(z, x) !\left(n(z, x)_{\mathrm{i}} 1\right) !\right.}{n(x) !}$ if $z, z+e^{i} 2 E_{i}(x)$ and $f_{i}^{s s}(z, x)=0$ otherwise, where $n(z, x)=\mathbf{j f} j 2 N \mathbf{j} z_{j}<x_{j} \mathrm{gj}$.

Example 3. The subsidy-free serial method $\varphi^{s f}$ (Moulin (1995)) averages the cost shares computed along the paths that follow the "constrained diagonal" to $x$. Let $G(x)=\mathbf{f}_{z} 2\left[0, x\left[\mathbf{j} 8 i, j 2 N,\left(x_{i} \cdot x_{j}\right)\right) \quad\left(\mathbf{j} z_{i} \mathbf{i} z_{j} \mathbf{j} \cdot 1\right.\right.$ or $\left.z_{i}=x_{i}<z_{j}\right) \mathbf{g}$ and $G^{\mathrm{a}}(x)=\mathbf{f} z 2\left[0, x\left[\mathbf{j} 8 i, j 2 N,\left(x_{i} \cdot x_{j}\right)\right) \quad\left(z_{i} \mathbf{i} \quad z_{j}=0\right.\right.$ or $\left.z_{i}=x_{i}<z_{j}\right)$ g. For each $z 2 G(x)$, denote by $\bar{z}(x)$ the smallest $z^{\alpha} 2 G^{\alpha}(x)$ such that $z^{\alpha}>z$ and let $\underline{z}(x)$ be the largest $z^{\natural} 2 G^{\natural}(x)$ such that $z^{\natural}$. $z$. The fow $f^{s f}(., x)$ associated with

\footnotetext{
${ }^{5}$ The original de.nition and characterizations of this method are in the model with continuous demands: Billera and Heath (1982), M irman and Tauman (1982). We present here its counterpart in the discrete model. For an axiomatization of[this discrete version, see Sprumont ( 2004).
} 
the serial method $\varphi^{s f}(., x)$ at $x$ is given by $f_{i}^{s f}(z, x)=f_{i}^{s s}(z \mathbf{i} \quad \underline{z}(x), \bar{z}(x) \mathbf{i} \quad \underline{z}(x))$ if $z, z+e^{i} 2 G(x)$ and $f_{i}^{s f}(z, x)=0$ otherwise.

We are now ready to describe the consequences of our four demand responsiveness conditions under full responsibility. First, we note that the two strongest conditions are incompatible with Additivity and Separability.

Proposition 2. Suppose $n$, 3. Then

i) no cost-sharing method satis..es Additivity, Separability, and Solidarity;

ii) no cost-sharing method satis..es Additivity, Separability, and Strong Group Monotonicity.

Proof. Statement i) We establish the incompatibility for $n=3$; the extension to $n, 3$ follows immediatel y by considering cost functions where all but three agents are dummies. Let $\varphi$ satisfy the three stated axioms and let $f$ be its $\ddagger$ ow representation as in formula (5). Let $x=e^{12}, x^{0}=e^{123}$. De.ne $C^{1} 2 \mathrm{C}$ by $C^{1}(z)=1$ if $z, e^{1}$ or $z, e^{23}$, and $C^{1}(z)=0$ otherwise. Similarly, de.ne $C^{2} 2 \mathrm{C}$ by $C^{2}(z)=1$ if $z, e^{2}$ or $z, e^{13}$, and $C^{2}(z)=0$ otherwise. Note that $C^{1}=D^{1}$ on $[0, x]$, where $D^{1}$ is the additively separable function $D^{1}(z)=1$ if $z_{1}, 1, D^{1}(z)=0$ otherwise. Thus by Independence of I rrelevant Costs and Separability $\varphi\left(C^{1}, x\right)=e^{1}$. On the other hand $\varphi_{2}\left(C^{1}, x 9\right)=f_{2}\left(e^{3}, x 9\right.$. A similar argument applied to $C^{2}$ gives $\varphi\left(C^{2}, x\right)=e^{2}$ and $\varphi_{1}\left(C^{2}, x\right)=f_{1}\left(e^{3}, x 9\right.$.

If $\varphi_{1}\left(C^{1}, x 9<\varphi_{1}\left(C^{1}, x\right)=1\right.$, Solidarity implies $\varphi_{2}\left(C^{1}, x 9 \cdot \varphi_{2}\left(C^{1}, x\right)=0\right.$, hence $\varphi_{2}\left(C^{1}, x^{9}\right)=0$. T he latter equality holds as well if $\varphi_{1}\left(C^{1}, x 9=\varphi_{1}\left(C^{1}, x\right)=1\right.$. Thus $f_{2}\left(e^{3}, x 9=0\right.$. A similar argument involving $C^{2}$ instead of $C^{1}$ gives $f_{1}\left(e^{3}, x 9=0\right.$. Now łow conservation yields $f_{3}\left(0, x^{9}\right)=0$. By exchanging the roles of the agents, we get similarly $f_{i}(0, x 9=0$ for $i=1,2$, a contradiction to the de. nition of a conservative unit ‡ow.

Statement ii) The proof mimics that of statement $i)$ : the .r.st paragraph is unchanged; then apply Strong Group Monotonicity to $C^{1}$ (with $i=3$ and $S=\mathrm{f} 1,3 \mathrm{~g}$ ) to get $\varphi_{2}\left(C^{1}, x 9 \cdot \varphi_{2}\left(C^{1}, x\right)=0\right.$, hence $f_{2}\left(e^{3}, x 9=0\right.$, and to $C^{2}$ (with $i=3$ and $S=\mathrm{f} 2,3 \mathrm{~g})$ to get $f_{1}\left(e^{3}, x 9\right)=0$. The rest is unchanged.

By contrast to Proposition 2, Monotonicity and Group M onotonicity are compatible with Additivity and Separability. Both have a lot of bite. As mentioned in the Introduction, the Aumann-Shapley method is not monotonic ${ }^{6}$. The Shapley-Shubik method is monotonic, but not group-monotonic. We only prove the latter statement.

\footnotetext{
${ }^{6} \mathrm{~A}$ simple counterexample has two agents and the cost function $\delta^{(1,1)}(z)=1$ for all $z,(1,1)$ and $\delta^{(1,1)}(z)=0$ otherwise. Then $\varphi^{a s}\left(\delta^{(1,1)},(1,1)\right)=\left(\frac{1}{2}, \frac{1}{2}\right)$ and $\varphi^{a s}\left(\delta^{(1,1)},(2,1)\right)=\left(\frac{1}{3}, \frac{2}{3}\right)$, a contradiction to Monotonicity.
} 
Let $N=\mathrm{f} 1,2,3 \mathrm{~g}$ and $C$ be the cost function:

$$
\begin{aligned}
C(z)= & 1 \text { if } z,(2,0,1) \text { or } z,(1,1,1) \text { or } z,(0,2,1), \\
& 0 \text { otherwise. }
\end{aligned}
$$

Check that $\varphi_{1}^{s s}(C,(1,1,1))=\varphi_{2}^{s s}(C,(1,1,1))=\frac{1}{3}>\frac{1}{6}=\varphi_{1}^{s s}(C,(2,2,1))=\varphi_{2}^{s s}(C$, $(2,2,1))$, which contravenes Group Monotonicity.

The characterization of all methods meeting Additivity, Separability and Group Monotonicity remains an open problem. In the rest of this section, we construct a fairly rich family of such methods.

We start with the ..xed-path methods. Any ..xed sequence $\pi:$ Nnfog ! $N$ generates an in. nite ..xed path $z^{\pi}: \mathbf{N} ! \mathbf{N}^{N}$ through the induction $z^{\pi}(0)=0, z^{\pi}(t)=$ $z^{\pi}(t ; \quad 1)+e^{\pi(t)}$ for $t 2 \mathrm{Nnf} 0 \mathrm{~g}$. If $\pi^{\mathrm{i}^{1}}(i)$ is in...nite for each $i 2 \mathrm{~N}$, we call the sequence $\pi$ unbounded; the corresponding ..xed path is unbounded in each coordinate (i.e., $z_{i}^{\pi}(t)=\mathbf{j f} s 2 \pi^{\mathrm{i}}{ }^{1}(i) \mathbf{j} s \cdot t \mathrm{gj}$ goes to in..nity as $t$ grows). To any demand pro..le $x 2$ $\mathrm{N}^{N}$, we associate a sequence $\pi^{x}: \mathrm{f} 1, \ldots, x(N) \mathrm{g} ! N$ by keeping the ..rst $x_{i}$ occurrences of each $i$ : for instance, if $n=3, x=(1,4,2)$ and $\pi=1,2,3,1,2,3,1,2,3, \ldots$, then $\pi^{x}=1,2,3,2,3,2,2$. The sequence $\pi^{x}$ in turn generates the monotone path $z^{\pi^{x}}$ to $x$ and the associated cost-sharing method $\varphi^{\pi^{x}}(., x)$ at $x$ via formula (6). The ..xed-path method $\varphi^{\pi}$ based on $\pi$ is de. ned by $\varphi^{\pi}(., x)=\varphi^{\pi^{x}}(., x)$ for all $x$.

To see why such methods satisfy G roup M onotonicity, ...X $\pi$ and $x$. For each $i 2 \mathrm{~N}$ let $t_{i}(\pi, x)$ be the smallest integer $t$ such that $z_{i}^{\pi}(t)=x_{i}$. Let $S \mu N$ and consider a demand pro..le $x^{0}$ such that $x_{i}<x_{i}^{0}$ for all $i 2 S$ and $x_{i}=x_{i}^{0}$ for all $i 2 \mathrm{NnS}$. Find the (necessarily unique) agent $i_{0} 2 S$ such that $t_{i_{0}}(\pi, x) \cdot t_{i}(\pi, x)$ for all $i 2 S$. Because $\pi$ is a ..xed sequence, $\varphi_{i_{0}}^{\pi}\left(C, x 9\right.$ is computed al ong a path $z^{\pi^{x^{0}}}$ to $x^{0}$ which coincides with $z^{\pi^{x}}$ for all $t \cdot t_{i_{0}}(\pi, x) \cdot t_{i_{0}}\left(\pi, x 9\right.$. Therefore $\varphi_{i_{0}}^{\pi}(C, x) \cdot \varphi_{i_{0}}^{\pi}(C, x)$, proving Group Monotonicity.

Fixed-path methods are asymmetric. To restore symmetry, we could take convex combinations of such methods, but the example of the Shapley-Shubik method demonstrates that in general this operation will not preserve Group M onotonicity. Interestingly, taking convex combinations of "suc ciently close" ..xed-path methods does preserve Group Monotonicity. Formally, let $\pi$ be a ..xed unbounded sequence and let $i, j 2 N$. Construct $\pi^{i j}: \mathbf{N n f} 0 \mathrm{~g} ! \mathbf{f} i, j \mathbf{g}$ by deleting from $\pi$ all occurrences of agents in $N \mathrm{nf} i, j \mathrm{~g}$. This sequence generates the projection of $z^{\pi}$ on $\mathrm{N}^{\mathrm{f} i, j \mathrm{~g}}$, denoted $z^{\pi^{i j}}: \mathrm{Nnf} 0 \mathrm{~g} ! \mathrm{N}^{\mathrm{f} i, j \mathrm{~g}}$, in the usual way. We say that two ..xed unbounded sequences $\pi, \sigma$ are nearby if for all $i, j 2 N$ and all $t 2 \mathrm{~N}$,

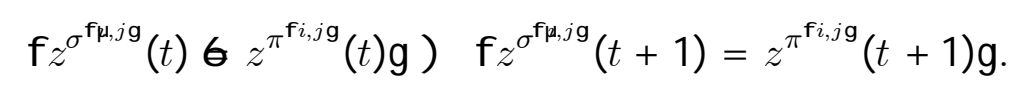


Proposition 3. If $\mathrm{f} \pi_{1}, \ldots, \pi_{K} \mathrm{gis}$ a family of unbounded sequences which pre pairwise nearby, and $\lambda_{1}, \ldots, \lambda_{K}$ are nonnegative numbers summing up to 1 , then ${ }_{k=1}^{K} \lambda_{k} \varphi^{\pi_{k}}$ satis..es Additivity, Separability, and G roup Monotonicity. In particular, the subsidyfree serial method (Example 3) satis..es these three axioms.

Proof. By Lemma 1, ${ }_{k=1}^{K} \lambda_{k} \varphi^{\pi_{k}}$ meets A dditivity and Separability. To check G roup Monotonicity, ... $C 2 C, S \mu N$, and $x, x^{0} 2 \mathrm{~N}^{N}$ such that $x_{i}<x_{i}^{0}$ for all $i 2 S$ and $x_{i}=x_{i}^{0}$ for all $i 2 \mathrm{NnS}$. Let $i_{1} 2 S$ be the agent in $S$ such that $t_{i_{1}}\left(\pi_{1}, x\right) \cdot t_{i}\left(\pi_{1}, x\right)$ for all $i 2 S$. As explained previously, $\varphi_{i_{1}}^{\pi_{1}}(C, x) \cdot \varphi_{i_{1}}^{\pi_{1}}(C, x 9$.

Next we show that $\varphi_{i_{1}}^{\pi_{k}}(C, x) \cdot \varphi_{i_{1}}^{\pi_{k}}(C, x 9$ for all $k 2 \mathrm{f} 2, \ldots, K \mathrm{~g}$. Fix $k$ and recall that $\pi_{k}$ is nearby $\pi_{1}$. This implies that for all $i 2 N$, either $t_{i_{1}}\left(\pi_{k}, x\right) \cdot t_{i}\left(\pi_{k}, x\right)$ or, if the opposite strict inequality is true,

$$
\left.t_{i}\left(\pi_{k}, x\right)<s<t_{i_{1}}\left(\pi_{k}, x\right)\right) \quad s \quad \pi_{k}^{\mathbf{i}^{1}}(i) .
$$

If $i 2 S$, the above property implies $t_{i 1}\left(\pi_{k}, x\right)<t_{i}\left(\pi_{k}, x+e^{i}\right) \cdot t_{i}\left(\pi_{k}, x\right)$. It follows that $t_{i_{1}}\left(\pi_{k}, x\right)<t_{i}\left(\pi_{k}, x 9\right.$ for all $i 2 S$. Therefore $\varphi_{i_{1}}^{\pi_{k}}(C, x 9$ is computed along a path to $x^{0}$ which coincides, for all $t \cdot t_{i_{1}}\left(\pi_{k}, x\right)$, with the path to $x$ al ong which $\varphi_{i_{1}}^{\pi_{k}}(C, x)$ is computed.

It follows that the subsidy-fre serial method meets the three axioms because $\varphi^{s f}$ is simply the arithmetic average of the $n$ ! methods generated by the ..xed unbounded sequences $p(1), p(2), \ldots, p(n), p(1), p(2), \ldots, p(n), \ldots$ corresponding to the possible permutations $p$ on $N$.

Our companion paper, Moulin and Sprumont (2002), oxers a characterization of the subsidy-free serial method based on the property called Distributivity, and expressing for the composition of cost functions (with perfect substitute outputs) the same invariance as A dditivity does for their addition.

\section{The fixed-flow met hods}

W ithin the full responsibility theory, we explore now two axioms weakening, respectively, Strong Ranking and Solidarity. Separability is incompatiblewith Strong Ranking but is clearly compatible with the following weaker condition:

Ranking. For all $C 2 \mathrm{C}, x 2 \mathrm{~N}^{N}$, and $i, j 2 N, \mathrm{f} C$ is a symmetric function of all its variables and $\left.x_{i} \cdot x_{j} \mathrm{~g}\right) \quad \mathrm{f} \varphi_{i}(C, x) \cdot \varphi_{j}(C, x) \mathrm{g}$.

The restriction to symmetric cost functions is very natural: when $C$ is symmetric, any dixerence in cost shares must originate in dixerences in demands, hence the ranking of cost shares should follow that of individual demands. Examples 1, 2, 3 all satisfy Ranking.

Next, Separability is incompatible with Solidarity (Proposition 2), but it is consistent with a restricted version of that axiom. Solidarity is very demanding when all 
types of production externalities are allowed, thus it is natural to limit its application to those cases where externalities are clearly signed. For any $C 2 \mathrm{C}, i, j 2 \mathrm{~N}$, and $z 2 \mathbf{N}^{N}$, de. ne $\partial_{i j} C(z)=\partial_{i} C\left(z+e^{j}\right) \mathbf{i} \partial_{i} C(z)$. The subsets of submodular and supermodular cost functions, respectively, are $\mathrm{C}_{\text {sub }}=\mathrm{f} C 2 \mathrm{Cj} \partial_{i j} C \cdot 0$ for all distinct $i, j 2 \mathrm{Ng}$ and $\mathrm{C}_{\text {sup }}=\mathrm{f} C 2 \mathrm{Cj} \partial_{i j} C, 0$ for all distinct $i, j 2 \mathrm{Ng}$.

Submodular Solidarity. For any $C 2 \mathrm{C}_{\text {sub }}$, any $i 2 N$, and any $x, x^{0} 2 \mathbf{N}^{N}, \mathbf{f} x_{j}=x_{j}^{0}$ for all $j 2 N \operatorname{nf} i \mathrm{gg}) \quad \mathrm{f} \varphi_{j}(C, x) \cdot \varphi_{j}\left(C, x 9\right.$ for all $j 2 N$ nf $i \mathrm{~g}$ or $\varphi_{j}(C, x), \varphi_{j}(C, x \varphi$ for all $j 2 \mathrm{Nnf} i \mathrm{gg}$. Supermodular Solidarity is de. ned by replacing $\mathrm{C}_{\text {sub }}$ with $\mathrm{C}_{\text {sup }}$ in the previous statement.

Examples 1, 2, 3 all satisfy the restricted solidarity properties. Combining either of them with Monotonicity and Separability circumscribes a very natural family of methods. For any $\ddagger$ ow $f(., x)$ on $\left[0, x\left[\right.\right.$ and any $x^{0} 2[0, x]$, the projection of $f(., x)$ on [0,x 9 , denoted $p_{x} \circ f(., x)$, is de..ned as follows: for any $i 2 N$ and $z 2[0, x q$ write $K=\mathbf{f} j 2 N \mathbf{j} z_{j}=x_{j}^{0} \mathbf{g}$ and let

$$
\begin{aligned}
p_{x^{0} f_{i}}(z, x) & =0 \text { if } \chi^{2 K}, \\
& =\underset{w_{K} 2\left[x_{K}^{0}, x_{K}\right]}{f} f_{i}\left(\left(w_{K}, z_{N \mathrm{n} K}\right), x\right) \text { otherwise, }
\end{aligned}
$$

with the convention that the sum is simply $f_{i}(z, x)$ if $K=;$. Note that $p_{x^{0}} f(., x)$ is a fow to $x^{0}$.

For simplicity, we state our characterization result for bounded cost-sharing problems: we ..X $\bar{x} 2$ (N HAf Og) ${ }^{N}$ and speak of a cost-sharing method restricted to $[0, \bar{x}]$ if $x$ varies in $[0, \bar{x}]$. The translation of all our axioms into this framework is straightforward: one merely needs to restrict their application to the demand pro. les in $[0, \bar{x}]$.

De..nition 2. A cost-sharing method $\varphi$ restricted to $[0, \bar{x}]$ is a ..xed- $\neq$ ow method if there is a fow $f(., \bar{x})$ to $\bar{x}$ such that, for every $x 2[0, \bar{x}]$, the $\neq$ ow $p_{x} f(., \bar{x})$ represents $\varphi(., x)$.

Examples include the..xed-path, Shapley-Shubik, and subsidy-free serial methods. One can check directly $f(., x)=p_{x} f\left(., x 9\right.$ for any $x, x^{0}, x \cdot x^{0}$, or apply our next result. On the other hand, the A umann-Shapley method is not a ..xed-łow method. The proof of the following result is in the A ppendix.

Theorem 2. Let $\bar{x} 2(\mathrm{NHf} 0 \mathrm{~g})^{N}$. For any cost-sharing method $\varphi$ restricted to $[0, \bar{x}]$, the following statements are equivalent:

i) $\varphi$ is a ..xed- $\ddagger$ ow method;

ii) $\varphi$ satis..es Additivity, Separability, M onotonicity, and Submodular Solidarity;

iii) $\varphi$ satis..es Additivity, Separability, Monotonicity, and Supermodular Solidarity. 
We conclude this section with three comments.

1) Theorem 2 is tight. For a method satisfying all axioms but Additivity, let

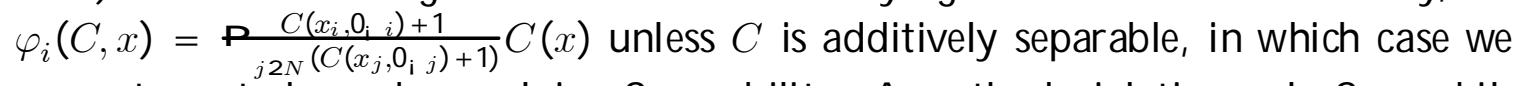
compute cost shares by applying Separability. A method violating only Separability is the proportional method $\varphi_{i}(C, x)=\frac{x_{i}}{x(N)} C(x)$. An example violating only Monotonicity is the Aumann-Shapley method: we prove in Section 6 that it actually satis. es properties stronger than Submodular Solidarity and Supermodular Solidarity. F inally, we describe a method violating only the latter two axioms. Let $n=3$ and $\bar{x}=(2,2,2)$. Consider the two monotone paths to $\bar{x}$ de..ned by the sequences

$$
\begin{aligned}
& \pi_{1}=1,2,3,1,2,3, \\
& \pi_{2}=1,2,3,2,1,3 .
\end{aligned}
$$

Let $f^{1}, f^{2}$ be the fows to $\bar{x}$ corresponding to $\pi_{1}, \pi_{2}$. For each $\left.x 2\right] 0, \bar{x}$ ], de. ne the fow $f(., x)$ to $x$ by $f(., x)=p_{x} f^{1}$ if $x=(2,2,1)$, and $f(., x)=p_{x} f^{2}$ otherwise. This generates a method restricted to $[0, \bar{x}]$ with the desired properties.

2) Fixed łow methods satisfy a stronger property than Dummy, called Strong Dummy: for all $C 2 \mathrm{C}, x 2 \mathrm{~N}^{N}$, and $\left.i 2 N, \mathbf{f} \partial_{i} C=0 \mathrm{~g}\right) \quad \mathbf{f} \varphi_{i}(C, x)=0$ and $\varphi_{j}(C, x)=\varphi_{j}\left(C,\left(0_{i}, x_{i}\right)\right)$ for all $j 2 \mathrm{Nnig}$. This follows by applying $f(., x 9=$ $p_{x^{0}} f(., x)$ to $x^{0}=\left(0_{i}, x_{i}\right)$. However the combination of Additivity, Strong Dummy and $M$ onotonicity is not enough to characterize the ..xed $\neq$ ow methods.

3) Theorem 2 suggests interesting open questions. Many ..xed-łow methods, including the Shapley-Shubik and subsidy-free serial methods, meet Ranking. If $n=2$, all ..xed-łow methods on $[0, \bar{x}]$ where $\overline{x_{1}}=\overline{x_{2}}$ and the fow $f$ is symmetric $\left(f_{1}(z, \bar{x})=f_{2}\left(\left(z_{2}, z_{1}\right), \bar{x}\right)\right.$ for all $\left.z\right)$ meet Ranking. Surprisingly, this property does not generalize to $n, 3$. Here is an example of a method based on a symmetric łow to $\bar{x}=\overline{x_{1}} e^{N}$ that violates Ranking. Let $n=3, \bar{x}=(3,3,3)$, and consider the six monotone paths to $\bar{x}$ de..ned by the sequence

$$
1,2,1,2,1,3,2,3,3
$$

and the ...ve sequences obtained from it by permuting agents. Let $f$ be the (fully symmetric) ‡ow on $[0, \bar{x}]$ obtained by putting an equal weight on these six paths, and let $\varphi$ be the resulting ..xed- $¥$ ow method. Consider the cost function

$$
\begin{aligned}
C(z)= & 1 \text { if } z, z^{\natural} \text { for some } z^{\natural} 2 Z^{\natural}, \\
& 0 \text { otherwise, }
\end{aligned}
$$

where $Z^{\mathfrak{\alpha}}=\mathrm{f}(1,2,2),(2,1,2),(2,2,1),(3,1,1),(1,3,1),(1,1,3) \mathrm{g}$. The function $C$ is symmetric in all its variables, and one computes $\varphi(C,(3,2,1))=\left(\frac{1}{2}, \frac{1}{6}, \frac{1}{3}\right)$, in contradiction of Ranking. This raises the problem of determining which ..xed- $\neq$ ow methods meet Ranking. 
Characterizing the class of ..xed-łow methods meeting Group Monotonicity is another challenging open problem.

6. Comparing the partial and full responsibil it y approaches Under Submodular or Supermodular Solidarity, the impact of an increase in agent $i$ 's demand on $j$ 's cost share must go in the same direction for all $j$ other than $i$ but this direction is a priori unrestricted. It is natural to require more: that all $j$ other than $i$ bene..t when costs are submodular, and suxer when costs are supermodular.

Positive Externalities. For any $C 2 \mathrm{C}_{\text {sub }}$, any $i 2 N$, and any $x, x^{0} 2 \mathbf{N}^{N}, \mathbf{f} x_{i}<x_{i}^{0}$ and $x_{j}=x_{j}^{0}$ for all $\left.j 2 N \operatorname{nf} i g g\right) \quad \mathrm{f} \varphi_{j}(C, x), \varphi_{j}(C, x 9$ for all $j 2 N$ nf $i \mathrm{gg}$. Negative Externalities is de. ned by replacing the subset $\mathrm{C}_{\text {sub }}$ with $\mathrm{C}_{\text {sup }}$ and the inequality sign, with - in the previous statement.

All quasi-proportional methods violate Positive Externalities and all but one violate Negative Externalities. The tension between these two axioms and Strong Ranking is systematic.

Proposition 4.

i) No cost-sharing method satis..es Strong Ranking and Positive Externalities.

ii) The only cost-sharing method satisfying Additivity, Strong Ranking and Negative Externalities is the egalitarian method ${ }^{7}$.

Proof. i) Consider the cost function $C(z)=0$ if $z \cdot 2 e^{1},=1$ otherwise. Check it is submodular (it is a particular case of the function de. ned by (26) in the A ppendix). By nonnegativity of cost shares and Positive Externalities, $\varphi(C, z)=0$ and $\varphi(C, z+$ $\left.e^{2}\right)=e^{2}$, violating Strong R anking. Note that A dditivity is not invoked.

ii) We focus on the case $n=2$ and leave the extension to the reader. Let $z \in 0$ and consider the supermodular cost function $\delta^{z}$ de. .ned by (28) in Step 3 of the proof of Theorem 2. Let $x$ be an arbitrary demand pro..le. If $\delta^{z}(x)=0$, the nonnegativity of cost shares forces $\varphi\left(\delta^{z}, x\right)=(0,0)$. If $\delta^{z}(x)=1$, distinguish three cases. If $x_{1}=x_{2}$, Strong R anking forces $\varphi\left(\delta^{z}, x\right)=\left(\frac{1}{2}, \frac{1}{2}\right)$. If $x_{1}<x_{2}$, Negative Externalities and the fact that $\varphi\left(\delta^{z},\left(x_{2}, x_{2}\right)\right)=\left(\frac{1}{2}, \frac{1}{2}\right)$ imply $\varphi_{2}\left(\delta^{z}, x\right) \cdot \frac{1}{2}$. Strong Ranking then forces $\varphi\left(\delta^{z}, x\right)=\left(\frac{1}{2}, \frac{1}{2}\right)$. A symmetrical argument handles the case $x_{1}>x_{2}$. This shows that $\varphi$ is the egalitarian method on the cost functions $\delta^{z}$. As every cost function coincides on every $[0, x]$ with a linear combination of such functions, the claim follows by Additivity.

By contrast, the ..xed- $¥$ ow methods satisfy Positive and Negative Externalities. Many more separable methods meet these axioms: an important example is the A umann-Shapley method. The proof of the following result is in the Appendix.

\footnotetext{
${ }^{7} \mathrm{~N}$ ote that Negative Externalities is implied by Full Solidarity and compare the current result with observation (4) following Theorem 1.
} 
Proposition 5. The ..xed-ł ow methods and the Aumann-Shapley method satisfy Positive Externalities and N egative Externalities.

To sum up, Strong Ranking is compatible with Solidarity but the direction of the cross-exects may be counter-intuitive; Separability is consistent with a limited form of solidarity only but the cross-exects may be guaranteed to have the expected sign.

7. Weak separabil it $y$

This section takes a second look at the partial responsibility theory. In M oulin and Sprumont (2002) we argue that a sound theory of partial responsibility should perform cross-subsidization to correct for cost asymmetries, and for that purpose only. Subsidization is not justi..ed when the cost function is symmetric: in such cases, the separability principle still applies. Thus if the cost function is not only symmetric but also additively separable, each agent will pay her "own" separable cost:

Weak Separability. For all $C 2 \mathrm{C}, x \mathbf{2} \mathrm{N}^{N}, \mathrm{f} C(z)={ }^{\mathrm{P}}{ }_{i 2 N} c\left(z_{i}\right)$ for all $\left.z \mathbf{2} \mathrm{N}^{N} \mathrm{~g}\right)$ $\mathbf{f} \varphi_{i}(C, x)=c\left(x_{i}\right)$ for all $i 2 \mathrm{Ng}$.

All quasi-proportional methods violate Weak Separability. For instance, if $n=2$, $x_{1}<x_{2}$, and $C(z)=c\left(z_{1}\right)+c\left(z_{2}\right)$, the proportional method cross-subsidizes agent 2 at 1 's expense when $c$ is convex, and vice-versa when $c$ is concave.

Y et, Weak Separability is compatible with Strong Ranking. A complete description of the methods meeting Additivity, Weak Separability, and Strong Ranking is oxered in Lemma 3 in the Appendix. A key member of that class is the crosssubsidizing serial method de..ned in Sprumont (1998) ${ }^{8}$.

Example 5. For any $x 2 \mathbf{N}_{x}^{N}=\mathbf{f} x 2 \mathbf{N}^{N}: x_{1} \cdot \ldots \cdot x_{n} \mathbf{g}$ and all $i 2 N$, de.ne $x^{i}=x_{i} e^{N} \wedge x$. The cross-subsidizing serial method $\varphi^{c s}$ assigns to every problem $(C, x) 2$ Cf $\mathrm{N}_{\mathfrak{x}}^{N}$ the vector of cost shares $\varphi^{c s}(C, x)=\frac{1}{n} C\left(x^{1}\right) e^{N}+\frac{1}{n_{\mathrm{i}} 1}\left[C\left(x^{2}\right) \mathbf{i}\right.$ $\left.C\left(x^{1}\right)\right] e^{N \mathrm{n} 1}+\ldots+\left[C\left(x^{n}\right)\right.$; $\left.C\left(x^{n^{1}}{ }^{1}\right)\right] e^{n}$. The cost shares for an arbitrary problem obtain by applying the formula after reordering the coordinates of the demand pro..le in nondecreasing order.

Proposition 6. The cross-subsidizing serial method satis..es Strong Ranking, W eak Separability, and Group Monotonicity. It fails Solidarity and Strong Group Monotonicity.

We omit the easy pro of of these two statements. The violation of Strong Group Monot onicity is a consequence of a general incompatibility recorded in the next proposition; see the A ppendix for a proof.

\footnotetext{
${ }^{8}$ See M oulin and Sprumont (2002) for a characterization based on the Distributivity axiom mentioned at the end of Section 4.
} 
Proposition 7. If $n, 3$, no cost-sharing method satis..es Additivity, Strong Ranking, Weak Separability, and Strong Group Monotonicity.

We conjecture that a similar incompatibility holds when Strong Group M onotonicity is replaced with Solidarity.

\section{Ref er ences}

[1] R. J . Aumann and L. Shapley, Values of Nonatomic Games, Princeton Univ. P ress, Princeton, NJ , 1974.

[2] W. Baumol, Panzar and R. Willig, Contestable Markets and the Theory of Industry Structure, Harcourt Brace J anovitch, New Y ork, 1982.

[3] L. Billera and D. Heath, A llocation of shared costs: A set of axioms yielding a unique procedure, Math. Oper. Res. 7 (1982), 32-39.

[4] E. Friedman and H. Moulin, Three methods to share joint costs or surplus, J. E con. Theory 87 (1999), 275-312.

[5] M. Haviv, TheA umann-Shapley price mechanism for allocating congestion costs, O per. Res. Letters 29 (2001), 211-215.

[6] C. Herrero, M. M aschler and A. Villar, Individual rights and collective responsibility: the rights-egalitarian solution, Math. Soc. Sci. 37 (1999), 59-77.

[7] D. Lee, Game-theoretic procedures for determining pavement thickness and traf... lane costs in highway cost allocation, Ph. D. thesis, Texas A\&M U niversity, 2002.

[8] L. Mirman and $Y$. Tauman, Demand compatible equitable cost sharing prices, Math. Oper. Res. 7 (1982), 40-56.

[9] H. Moulin, On additive methods to share joint costs, Japan. Econ. Rev. 46 (1995), 303-332.

[10] H. M oulin and Y. Sprumont, Responsibility and cross-subsidization in cost sharing, mimeo (2002).

[11] H. Moulin and R. Vohra, A representation of additive cost sharing methods, E con. Letters, 80 (2003), 399-407.

[12] N. Naumova, Non symmetric equal sacri..ce solutions for cost allocation problems, Math. Soc. Sci. 43 (2002), 1-18. 
[13] L. Shapley, A value for $n$-person games, in "Contributions to the Theory of Games II" (H.W. K uhn and W. Tucker, E ds.), Ann. Math. Stud. Vol. 28, Princeton Univ. Press, Princeton, NJ , 1953.

[14] W. Sharkey, The Theory of natural Monopoly, Cambridge Univ. Press, Cambridge, 1982.

[15] S. Shenker, Making greed work in networks: a game-theoretic analysis of gateway service discipline, IE EE / ACM Trans. Networking 3 (1995), 819-831.( circulated 1989).

[16] M. Shubik, Incentives, decentralized control, the assignment of joint costs, and internal pricing, Managt. Sci. 8 (1962), 325-343.

[17] Y . Sprumont, Ordinal cost sharing, J . Econ. Theory 81 (1998), 126-162.

[18] Y . Sprumont, A umann-Shapley pricing: a reconsideration of the discrete case, mimeo, Université de Montréal, 2004.

[19] Y Sprumont and L. Zhou, Pazner-Schmeidler rules in large societies, J . Math. E con. 31 (1999), 321.339.

[20] W. Thomson, Welfare-domination under preference replacement: a survey and open questions, Soc. Choice Welfare 16 (1999), 373-394.

[21] Y. Wang, The additivity and dummy axioms in the discrete cost sharing model, E con. Letters 64 (1999), 187-192.

[22] R. Weber, Probabilistic values for games, in "The Shapley Value" (A. Roth E d.), Cambridge Univ. Press, Cambridge, 1988.

\section{Appendix}

8.1. Shared-łow representation of additive methods. De..nea sharing rule for $x \mathbf{2} \mathbf{N}^{N}$ to be a mapping $s(., ., x):[0, x[\mathbf{f} N ! \phi$. The vector $r(z, j, x)$ speci..es how the $\neq$ ow $f_{j}(z, x)$ between $z$ and $z+e^{j}$ is shared among the agents. Choosing for each demand pro..le $x$ a fow $f(., x)$ and a sharing rule $r(., ., x)$, the formula

$$
\varphi(C, x)={ }_{z 2[0, x[j 2 N} \partial_{j} C(z) f_{j}(z, x) r(z, j, x)
$$

for all $C 2 \mathrm{C}$ de. nes an additive cost-sharing method. Keep in mind that $r$ is vectorvalued while $\partial_{j} C$ and $f_{j}$ are scalar-valued.

Conversely, Moulin and Vohra (2003) show that all additive methods possess at least one such shared- $\ddagger$ ow representation $(f, r)$. Observe that these methods meet Independence of Irrelevant C osts. 
8.2. Statement and proof of Theorem 1*. Theorem $1^{*}$ and Lemma 2 below constitute a more general characterization result than Theorem 1, in which we drop the Strong Ranking assumption. Denote by $R_{+}$and $R_{++}$the sets of nonnegative and strictly positive real numbers, respectively. For each $i 2 \mathrm{~N}$, choose a function $s_{i}: \mathrm{N} ! \mathrm{R}_{+}$such that $s_{i}\left(x_{i}\right)>0$ whenever $x_{i}>0$. If $x>0$, write $F_{i}(x)=\frac{p_{i}\left(x_{i}\right)}{N_{j}\left(x_{j}\right)}$ and $F(x)=\left(F_{1}(x), \ldots, F_{n}(x)\right) \mathrm{N}$ ext, choose a function $\mu_{i}$ assigning a real number to every pair $\left(x_{i}, t_{i}\right) 2(\mathrm{Nnf} 0 \mathrm{~g})^{2}$ such that $t_{i} \cdot x_{i}$. For every cost-sharing problem $(C, x) 2 C \in \mathrm{N}^{N}$ and each $i 2 N$, de. ne

$$
s a^{\mu_{i}}\left(C, x_{i}\right)={ }_{t_{i}=1}^{X_{i}} \mu_{i}\left(x_{i}, t_{i}\right) \partial_{i} C\left(\left(t_{i} \mathbf{i} 1\right) e^{i}\right),
$$

which may be viewed as a generalized stand-alone cost for agent $i$. The formula

$$
\varphi_{i}^{s, \mu}(C, x)=s a^{\mu_{i}}\left(C, x_{i}\right)+F_{i}(x)\left(C(x) \mathbf{i}_{j 2 N}^{\mathbf{X}} s a^{\mu_{j}}\left(C, x_{j}\right)\right)
$$

de. nes an additive cost-sharing method provided cost shares are always non negative. Setting $\alpha_{j}^{+}\left(x_{j}\right)=\operatorname{maxf} 0, \mu_{j}\left(x_{j}, t_{j}\right) \mathrm{j} t_{j} 2\left[1, x_{j}\right] \mathrm{g}$ and $\alpha_{j}^{\mathrm{i}}\left(x_{j}\right)=\operatorname{minf} 0, \mu_{j}\left(x_{j}, t_{j}\right) \mathrm{j} t_{j} 2$ $\left[1, x_{j}\right] \mathrm{g}$, this amounts to imposing the following restrictions on $f_{j}, \mu_{j}$ for all $i$ and $x$ :

$$
F_{i}(x)\left(1_{\mathbf{i}}{ }_{N \mathrm{n} i}^{\mathbf{X}} \alpha_{j}^{+}\left(x_{j}\right)\right)+\left(1_{\mathbf{i}} F_{i}(x)\right) \alpha_{i}^{\mathrm{i}}\left(x_{i}\right), 0 .
$$

This claim follows easily from the inequalities $\alpha_{i}^{\mathrm{i}}\left(x_{i}\right) C\left(x_{i}, 0_{\mathrm{i}} i\right) \cdot s a^{\mu_{i}}\left(C, x_{i}\right) \cdot$ $\alpha_{i}^{+}\left(x_{i}\right) C\left(x_{i}, 0_{\mathrm{i}} i\right)$; we omit the straightforward details. By construction, we have

Lemma 2. Every method $\varphi^{s, \mu}$ constructed as in (9) and (10) satis..es Solidarity. If $s_{i}(0)>0$ for all $i 2 N, \varphi^{s, \mu}$ also satis..es Strict Solidarity.

M onotonicity imposes further restrictions on $s, \mu$. One of them is that each $s_{i}$ is nondecreasing (to see this, consider in (9) a function $C$ such that $C\left(x_{i}, 0_{\mathrm{i}} i\right)=0$ for all $i$ ). Necessary and su $₫$ cient conditions seem diф cult to formulate in a simple way; in particular, the inequalities $\mu_{i}, 0$ are not implied. In the particular case where $\mu_{i}$ is independent of both $t_{i}$ and $x_{i}$, however, one checks that $\varphi^{s, \mu}$ meets Monotonicity if and only if $s_{i}$ is nondecreasing and $\mu_{i}, 0$ for all $i$. Then the inequalities (10) reduce to ${ }_{j 2 \mathrm{Nni}} \mu_{j} \cdot 1$ for all $i$. Adding the requirement of symmetry, we obtain the family of methods

$$
\varphi_{i}(C, x)=\frac{\lambda}{n \mathbf{i} 1} C\left(x_{i}, 0_{\mathbf{i}} i\right)+\frac{\mathbf{p} s\left(x_{i}\right)}{{ }_{N} s\left(x_{j}\right)}\left(C(x) \mathbf{i}{ }_{j 2 N}^{\mathbf{X}} \frac{\lambda}{n \mathbf{i} 1} C\left(x_{j}, 0_{\mathbf{i} j}\right)\right)
$$


where $0 \cdot \lambda \cdot 1$, and $s: \mathbf{N} ! \mathbf{R}_{+}$. When $s\left(x_{i}\right)=1$ for all $x_{i}$, this formula reduces to (4) in Section 3.

Theorem 1*.

i) A ssume $n, 4$ and let $\varphi$ be a cost-sharing method satisfying Additivity, Strict Solidarity, and Monotonicity. For all $i 2 \mathrm{~N}$, there exists a nondecreasing function $s_{i}: \mathbf{N} ! \mathbf{R}_{++}$and a real-valued function $\mu_{i}$ such that $\varphi=\varphi^{s, \mu}$, where $\varphi^{s, \mu}$ is de..ned in (9).

ii) Assume $n, 3$ and let $\varphi$ be a cost-sharing method satisfying Additivity, Solidarity, Monotonicity, Zero Cost for Zero Demand, and Positive Cost for Positive Demand. For all $i 2 N$ there exists a nondecreasing function $s_{i}: \mathbf{N} ! \mathbf{R}_{+}$with $s_{i}(0)=0<s_{i}(1)$, and a real-valued function $\mu_{i}$ such that $\varphi=\varphi^{s, \mu}$.

Proof of Theorem $1^{*}$, statement i)

We ..x throughout the pro of a method $\varphi$ meeting Additivity, Strict Solidarity, and Monotonicity. R ecall that $\mathrm{D}$ is the subset of cost functions $D$ such that $D(z)=0,1$. Writing $\partial D=\mathbf{f} z 2 D \mathbf{j} z \mathbf{i} e^{i} 2 D$ for all $i 2 N(z) \mathbf{g}$, Independence of Irrelevant Costs yields $\varphi(D, x)=\varphi\left(D^{0}, x\right)$ whenever $D, D^{0} 2 \mathrm{D}$ and $x 2 \partial D \backslash \partial D^{0}$. We may therefore de. ne $\theta: \mathbf{N}^{N}$ ! $\$$ by

$$
\theta(x)=\varphi(D, x),
$$

where $D$ is any cost function in D such that $x 2 \partial D$.

We spend Steps 1 to 5 computing $\varphi(D, x)$ for $D 2$ D. After the preliminary Steps 1 to 3 , we construct the desired functions $s_{i}, i=1, . . n$, and derive the quasiproportional form $\theta_{i}(x)=F_{i}(x)$ in Step 4. Step 5 computes $\varphi(D, x)$ for any $D, x$. Using Additivity, Step 6 then derives $\varphi(C, x)$ for all $C 2 \mathrm{C}$.

We use the following notation. If $D 2 \mathrm{D}$ and $x 2 \mathbf{N}^{N}, t_{i}\left(D, x_{\mathbf{i} i}\right)=\inf \mathbf{f} x_{i} \mathbf{j}\left(x_{i}, x_{\mathbf{i}}\right) 2$ $D \mathrm{~g}$, with the convention $t_{i}=+1$ if this set is empty. For any $S \mu N, \phi(S)=\mathbf{f} y 2$ $\mathrm{R}_{+}^{S} \mathrm{j} \quad{ }_{i 2 S} y_{i}=1 \mathrm{~g}$.

Step 1. We show that if $D 2 \mathrm{D}, x 2 D$ and there exist two distinct agents $i, j 2 N$ such that $t_{i}\left(D, x_{\mathbf{i} i}\right)>0$ and $t_{j}\left(D, x_{\mathbf{i} j}\right)>0$, then $\varphi(D, x) \grave{A} 0$. It follows that for any $\left.x \mathbf{2} \mathbf{N}^{N}, \mathbf{f} n(x), 2 \mathrm{~g}\right) \quad \mathbf{f} \theta(x) \grave{A} 0 \mathrm{~g}$.

To prove the ...rst statement, assume its premises and consider the increase in $i$ 's demand from 0 to $x_{i}$. By Strict Solidarity, $\varphi_{\mathrm{i} i}(D, x) \mathbf{i} \varphi_{\mathrm{i} i}\left(D,\left(x_{\mathrm{i} i}, 0_{i}\right)\right)$ is either strictly positive or zero. In the latter case, $\varphi(D, x)=e^{i}$, and in the former case $\varphi_{\mathrm{j} i}(D, x) \grave{A} 0$. Repeating this argument with agent $j$, either $\varphi(D, x)=e^{j}$ or $\varphi_{i}(D, x) \AA \quad 0$. The only possibility is therefore that $\varphi_{i}(D, x) \grave{\AA} 0$ and $\varphi_{i}(D, x) \grave{\AA} 0$, hence $\varphi(D, x) \grave{\AA} 0$. The second statement follows from the ...rst because $t_{i}\left(D, x_{\mathbf{i} i}\right)=x_{i}$ for all $i$ whenever $x 2 \partial D$. 
Step 2. We show that for any D $2 \mathrm{D}, i 2 N, x 2 D$ such that $x_{\mathrm{i} i} \in 0$, and $x^{0}=\left(x_{i}^{0}, x_{\mathrm{i} i}\right)$ such that $x_{i}^{0}>x_{i}$,

$$
\varphi_{\mathbf{i} i}(D, x)_{\mathbf{i}} \varphi_{\mathbf{i} i}(D, x)=\mathbf{i} \lambda \theta_{\mathbf{i} i}(x) \text { for some } \lambda, 0 .
$$

M onotonicity implies $\varphi_{i}\left(D, x 9 \mathbf{i} \varphi_{i}(D, x), 0\right.$, whereas ${ }^{\mathrm{P}}{ }_{N}\left(\varphi_{j}\left(D, x q_{\mathbf{i}} \varphi_{j}(D, x)\right)=\right.$ 0 by budget balance. Thus, by Solidarity,

$$
\varphi_{j}(D, x)_{\mathbf{i}} \varphi_{j}(D, x) \cdot 0 \text { for all } j \in i .
$$

Choose $D^{0} 2 \mathrm{D}$ such that $x^{0} 2 \partial D^{0}$ and let $\alpha 2 \mathbf{R}_{++}$. A pplying Strict Solidarity to $\alpha D+D^{0}$ when $x_{i}$ increases to $x_{i}^{0}$, we obtain that $\operatorname{signf} \alpha\left(\varphi_{j}\left(D, x 9_{\mathrm{i}} \varphi_{j}(D, x)\right)+\right.$ $\left(\varphi_{j}\left(D^{0}, x 9 \mathrm{i} \varphi_{j}\left(D^{0}, x\right)\right) \mathrm{g}\right.$ is independent of $j, j \in i$, where $\operatorname{sign}(u)$ is 1,0 , or $\mathbf{i} 1$ if $u$ is respectivel y positive, zero or negative. From Step $1, \varphi_{j}\left(D^{0}, x q_{\mathbf{i}} \varphi_{j}\left(D^{0}, x\right)=\theta_{j}(x 9>0\right.$ because $n(x), 2$. On the other hand $\varphi_{j}\left(D, x 9_{\mathbf{i}} \varphi_{j}(D, x)=u_{j} \cdot 0\right.$. By Strict Solidarity, $u_{\mathrm{i} i} i \quad 0$ or $u_{\mathrm{i} i}=0$. In the latter case, (11) holds with $\lambda=0$. In the former, the vector $\alpha u_{\mathrm{i} i}+\theta_{\mathrm{i} i}(x 9$ is either strictly positive, zero, or strictly negative. Since this holds for all $\alpha>0, u_{\mathrm{i} i}$ and $\theta_{\mathrm{i} i}(x 9$ are parallel and of opposite sign, and (11) holds with $\lambda>0$.

Step 3. We show that for any two distinct $i, j 2 N$ and any $x_{\mathrm{i} i j} \in 0$, the direction of $\theta_{\mathrm{i} i j}\left(y_{i j}, x_{\mathrm{i} i j}\right)$ is independent of $y_{i j} 2 \mathrm{~N}^{2} \mathrm{nf} 0 \mathrm{~g}$. That is, there exists $\rho\left(x_{\mathrm{i} i j}\right) 2$ $\$(N \mathrm{n} i j)$ such that, for all $y_{i j} 2 \mathrm{~N}^{2} \mathrm{nf} 0 \mathrm{~g}, \theta_{\mathrm{i} i j}\left(y_{i j}, x_{\mathrm{i} i j}\right)=\lambda \rho\left(x_{\mathrm{i} i j}\right)$ for some $\lambda>0$.

Take $i=1, j=2, . . \mathbf{x} x_{\mathrm{i} 12} \in 0$, and write $\theta_{\mathrm{i} 12}\left(y_{12}, x_{\mathrm{i} 12}\right)=s(y) 2 \mathbf{R}_{+}^{N n 12}$, where $y=\left(y_{1}, y_{2}\right) 2 \mathbf{N}^{2} \mathrm{nf} 0 \mathrm{~g}$. From now on, we do not repeat the restriction $y \boldsymbol{\sigma} 0$. B y Step $1, s(y) \AA$ A 0 .

Pick $y, y^{0}$ non-comparable (i.e., neither $y \cdot y^{0}$ nor $y^{0} \cdot y$ holds) and $D$ such that $\partial D$ contains $y$ and $y^{0}$. Applying (11) twice, respectively to the increase from $y$ to $y_{-} y^{0}$, and from $y^{0}$ to $y_{-} y^{0}$,

$$
\begin{aligned}
& \varphi_{\mathrm{i} 12}\left(D, y_{-} y \mathbf{9}_{\mathbf{i}} s(y)=\mathbf{i} \lambda s(y+y 9,\right. \\
& \varphi_{\mathrm{i} 12}\left(D, y_{-} y^{9} \mathbf{i} s(y)=\mathbf{i} \lambda^{0} s\left(y_{-} y{ }^{9} .\right.\right.
\end{aligned}
$$

Taking the dixerence of these equalities,

$$
s(y) \text { i } s\left(y 9=\mu s\left(y \_y 9 \text { for some } \mu 2 \mathrm{R} .\right.\right.
$$

This property holds for any non-comparable $y, y^{0}$.

Recall that our goal is to show that the direction of $s(y)$ is independent of $y$. To this end, assume ..rst that there exist $y, y^{0}$ with $y_{2}=y_{2}^{0}>0, y_{1}<y_{1}^{0}$ and $s(y) \in s(y 9$. 
Construct $z, z^{0}$ such that $z_{1}=z_{1}^{0}>y_{1}^{0}$ and $z_{2}<z_{2}^{0}=y_{2}^{0}$ (see Figure 1). Two applications of (13) give

$$
\begin{aligned}
s(z) \mathrm{i} s(y) & =\lambda s\left(z^{9},\right. \\
s(z) \mathrm{i} s(y) & =\lambda^{0} s\left(z^{9} .\right.
\end{aligned}
$$

Taking dixerences we..nd that $s(z 9$ and $s(y)$ i $s(y 9$ have the same direction $\rho, \rho \grave{A} \quad 0$. Let $L$ be the straight line borne by $\rho$ and containing $s(y)$. It contains $s(z)$ and $s(y 9$.

Now consider $w, w^{0}$ such that $w_{1}=w_{1}^{0}>z_{1}^{0}$ and $w_{2}<w_{2}^{0}=z_{2}^{0}$ (se Figure 1). By the above argument, $s\left(w^{9}\right)$ is borne by $\rho$, and $L$ contains $s(w)$. Applying (13) to $z^{0}, w$ gives

$$
s(z 9 \text { i } s(w)=\mu s(w 9 .
$$

Thus $s(z 9) 2 L$, implying that $L$ is the line borne by $\rho$ through 0 . Now $s$ is the direction of $s(t)$ for the six points $t$ of our construction.

We check next that $s(t)$ is borne by $\rho$ for any $t$ such that $t_{2} \cdot y_{2}$ (and $t \in 0$ ). We just proved this under the assumption that $t_{1}>y_{1}^{0}$. Next suppose $t_{1} \cdot y_{1}^{0}$ and $0<t_{2} \cdot y_{2}$. Set $z_{0}=\left(y_{1}^{0}+1,0\right)$ and $t^{0}=t_{-} z_{0}$ (see F igure 2). By (13) applied to $t, z_{0}, s(t)$ is borne by $\rho$. The remaining values of $t$ are $t=\left(t_{1}, 0\right)$ with $0<t_{1} \cdot y_{1}^{0}$. There we apply (13) to $t$ and $t^{0}=\left(t_{1}\right.$ i 1,1$)$.

Summing up, we have shown that if $s$ is not constant on a given horizontal line $\mathbf{f} y \mathbf{j} y_{2}=\varepsilon>0 \mathbf{g}$, then the direction of $s(y)$ is constant on the whole band $\mathbf{f} y \mathbf{j} y_{2} \cdot \varepsilon$; $y \in 0 \mathrm{~g}$. If there are such integers $\varepsilon$ as large as we want, this establishes the desired statement at once. The remaining case is when there is a number $\varepsilon$ such that $s$ is constant on every line $\mathrm{f} y \mathbf{j} y_{2}=\varepsilon^{0} \mathrm{~g}, \varepsilon^{0}, \varepsilon$. For any $y$ such that $y_{1}>0$, apply (13) to $y$ and $y^{0}=\left(y_{1} \mathrm{i} 1, \varepsilon 9\right)$ where $\varepsilon^{0}, \operatorname{maxf} y_{2}+1, \varepsilon \mathbf{g}$ : we deduce that $s(y)$ is borne by the value of $s$ on $\mathbf{f} y \mathbf{j} y_{2}=\varepsilon \mathrm{g}$. The remaining case $y_{1}=0$ is handled similarly.

Step 4. We show that there exist $n$ functions $s_{i}: \mathbf{N} ! \mathrm{R}_{++}$such that, for all $\left.x 2 \mathbf{N}^{N}, \mathbf{f} n(x), 2 \mathrm{~g}\right) \quad \mathbf{f} \theta(x)=F(x) \mathbf{g}$.

We begin by considering the demand pro..les $x$ such that $n(x)=n$. In particular, $\theta(x)$ À 0 . By Step 3, the ratio $\frac{\theta_{i}}{\theta_{j}}(x)$ is independent of $x_{k}$ for all $k \boldsymbol{\theta} i, j$ (recall $n, 4$ ). For $i, j, k$ all distinct,

$$
\left.\frac{\theta_{i}}{\theta_{j}}(x)=s_{i j}\left(x_{i}, x_{j}\right)\right) \quad s_{i j}\left(x_{i}, x_{j}\right) s_{j k}\left(x_{j}, x_{k}\right) s_{k i}\left(x_{k}, x_{i}\right)=1 .
$$

A standard argument (omitted for brevity), shows the existence of $n$ functions $s_{i}$ on Nnf Og such that $\frac{\theta_{i}}{\theta_{j}}(x)=\frac{s_{i}\left(x_{i}\right)}{s_{j}\left(x_{j}\right)}$ for all $i, j$, all $x$. Thus the desired form $\theta(x)=F(x)$ holds whenever $n(x)=n$. 
Next we treat the case $n(x)=n \mathbf{i} 1$, de.ning $s_{i}(0)$ in the process. Consider some $x$ with $N(x)=N \mathrm{nl}$. For all $i, j, 2$, we have

$$
\frac{\theta_{i}}{\theta_{j}}(x)=\frac{\theta_{i}}{\theta_{j}}\left(1, x_{\mathrm{i}} 1\right)=\frac{s_{i}\left(x_{i}\right)}{s_{j}\left(x_{j}\right)},
$$

where the ..rst equality follows from Step 3. By Step 3 again, $\frac{\theta_{1}}{\theta_{2}}(x)$ does not depend upon $x_{j}$, for any $j \in 1,2$, provided $x_{j}$ remains positive:

$$
\frac{\theta_{1}}{\theta_{2}}(x)=\frac{\theta_{1}}{\theta_{2}}\left(0, x_{2}, 1, \ldots 1\right)=: \frac{1}{g_{2}\left(x_{2}\right)} .
$$

De.ning $g_{i}$ for $i=3, \ldots, n$ similarly and combining the two properties above,

$$
\frac{s_{i}\left(x_{i}\right)}{s_{j}\left(x_{j}\right)}=\frac{g_{i}\left(x_{i}\right)}{g_{j}\left(x_{j}\right)} \text { for all } i, j \in 1 \text { and all } x_{\mathrm{i} 1} \AA \text { А } 0 \text {. }
$$

Thus $\frac{s_{i}}{g_{i}}$ depends neither on $i$ nor on $x_{i}$. Calling this ratio $s_{1}(0)$ we now have $\frac{\theta_{i}}{\theta_{j}}(x)=$ $\frac{s_{i}\left(x_{i}\right)}{s_{j}\left(x_{j}\right)}$ for all $i, j$. A similar construction delivers $s_{i}(0)$ for all $i$, and proves $\theta(x)=F(x)$ when $n(x)=n \mathbf{i} 1$.

We complete the argument by decreasing induction on $n(x)$. Fix $q, 2 \cdot q \cdot n_{\mathbf{i}} 2$. A ssume $\theta(x)=F(x)$ whenever $n(x), q+1$ and consider $x$ with $n(x)=q$, say $N(x)=$ $\mathrm{f} 1, \ldots, q \mathrm{~g}$. For any distinct $i, j 2 \mathrm{N \textrm {nf }} \mathbf{1}, n \mathrm{~g}$, Step 3 and the inductive assumption imply

$$
\frac{\theta_{i}}{\theta_{j}}(x)=\frac{\theta_{i}}{\theta_{j}}\left(x_{\mathrm{i} n}, 1_{n}\right)=\frac{s_{i}\left(x_{i}\right)}{s_{j}\left(x_{j}\right)} .
$$

Note that $q, 2$ ensures $x_{\mathbf{i} i, j} \in 0$, as required to apply Step 3. Moreover, $q \cdot n \mathbf{i} 2$ guarantees that we have at least two choices for agent 1 in $N(x)$, ditto for agent $n$ in $N \mathrm{n} N(x)$. The equality $\theta(x)=F(x)$ is now clear.

Step 5. We derive an explicit formula for $\varphi(D, x)$ for all $D 2 \mathrm{D}$ and $x 2 D$. U sing the notation $\tau_{i}(D)=t_{i}\left(D, 0_{\mathrm{i} i}\right)$, note that $x_{i} e^{i} 2 D, \tau_{i}(D) \cdot x_{i}$ and de.ne $K(D, x)=\mathbf{f} i 2 N \mathbf{j} \tau_{i}(D) \cdot x_{i} \mathbf{g}$. De.ne $H: \mathbf{R}_{+}^{N} \operatorname{nf} 0 \mathrm{~g} f \mathbf{R}^{N} ! \mathbf{R}^{N}$ by

$$
H_{i}(a, b)=\frac{\mathrm{p} a_{i}}{{ }_{N} a_{j}}\left(1+{ }_{N \mathrm{n} i}^{\mathrm{X}}\left(b_{i} \mathbf{i} b_{j}\right) a_{j}\right) .
$$

We show that there exist $n$ functions $\lambda_{i}$ such that, for all $D 2 \mathrm{D}$ and $x 2 D$,

$$
\begin{aligned}
\varphi(D, x)= & H(a, b), \text { where for all } i \\
a_{i}= & s_{i}\left(x_{i}\right), \\
b_{i}= & \lambda_{i}\left(x_{i}, \tau_{i}(D)\right) \text { if } i 2 K(D, x), \\
& 0 \text { otherwise. }
\end{aligned}
$$


In Step 5.a, we prove (15) when $K(D, x)=$ ?. In Step 5.b, we construct the functions $\lambda_{i}$ and prove (15) when $\mathrm{j} K(D, x) \mathrm{j}=1$. Step $5 . c$ completes the proof by an induction argument on $\mathbf{j} K(D, x) \mathbf{j}$. $z 2 \mathrm{R}^{N}$,

T wo simple facts about $H$ will be useful. First ${ }^{\mathrm{P}}{ }_{N} H_{i}(a, b)=1$. Next, for any

$$
\begin{gathered}
\mathrm{f}_{N}^{\mathrm{X}} z_{i}=1 \text { and } \frac{z_{i}}{a_{i}} \mathrm{i} \frac{z_{j}}{a_{j}}=b_{i} \mathbf{i} b_{j} \text { for all } i, j \text { such that } a_{i}, a_{j} \in 0 \mathrm{~g} \\
\text { ) } \quad \mathbf{f} z=H(a, b) \mathbf{g} .
\end{gathered}
$$

We will use the notation $x^{\mathrm{i} i}=\left(0_{i}, x_{\mathrm{i} i},\right)$.

Step 5.a. When $K(D, x)=$ ?, (15) reduces to $\varphi(D, x)=F(x)$. We prove this equality by induction on $n(x)$.

The smallest possible size is $n(x)=2$ : if $x=x_{1} e^{1}$, say, then $x 2 D$ forces $\tau_{1}(D) \cdot x_{1}$. So we ..x $x$ with $N(x)=\mathbf{f} 1,2 \mathrm{~g}$. De..ne $x_{\ltimes}=x+e^{3}$ and choose $D^{\mathfrak{\alpha}}$ such that

$$
D^{\mathfrak{\alpha}} \backslash[0, x]=D \backslash[0, x] \text { and } x_{\alpha}^{\mathrm{i} i} 2 \partial D^{\mathfrak{\alpha}} \text { for } i=1,2 .
$$

This is possible because $x_{\alpha}^{\mathrm{i}}{ }^{1} \mathbf{i} e^{3}=x^{\mathrm{i}}{ }^{1}=x_{2} e^{2} 2 D$ and similarly $x_{\mathrm{a}}^{\mathrm{i}}{ }^{2} \mathbf{i} e^{3} 2 D$.

By construction of $D^{\alpha}$ and Step $4, \varphi\left(D^{\alpha}, x_{\alpha}^{\mathrm{i}}{ }^{1}\right)=\theta\left(x_{\alpha}^{\mathrm{i}}{ }^{1}\right)=F\left(x_{\alpha}^{\mathrm{i}}{ }^{1}\right)$. Applying (11) to $D^{\mathfrak{\alpha}}, x_{\ltimes}$ and $x_{\mathfrak{\alpha}}^{\mathrm{i}} 1$,

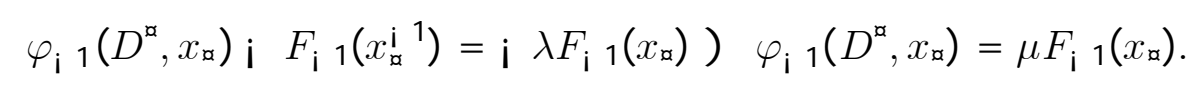

This, the symmetric property upon exchanging 1 and 2 , and the fact that $F\left(x_{\mathrm{a}}\right) \grave{\mathrm{A}} 0$, give. . nally $\varphi\left(D^{\mathrm{a}}, x_{\mathrm{a}}\right)=F\left(x_{\mathrm{a}}\right)$.

Next we apply (11) to $D^{\mathfrak{a}}, x_{x}$ and $x_{\alpha}^{\mathrm{i}}{ }^{3}=x$ :

$$
\varphi_{\mathrm{i} 3}\left(D^{\natural}, x_{\mathrm{a}}\right) \mathbf{i} \varphi_{\mathbf{i} 3}\left(D^{\natural}, x\right)=\mathbf{i} \lambda F_{\mathbf{i} 3}\left(x_{\mathrm{a}}\right)=\mathbf{i} \lambda^{0} F_{\mathbf{i}} 3(x) .
$$

Independence of I rrelevant Costs implies $\varphi\left(D^{\natural}, x\right)=\varphi(D, x)$, so we conclude $\varphi_{i 3}(D, x)$ $=\mu F_{\mathrm{i}} 3(x)$ for some $\mu, 0$. R epeating this construction with coordinate 4 instead of 3 yields $\varphi_{\mathrm{i}} 4(D, x)=\nu F_{\mathrm{i}} 4(x)$ for some $\nu, 0$. The conclusion $\varphi(D, x)=F(x)$ follows as in the previous paragraph.

Now the induction argument. Fix $D, x$ such that $K(D, x)=$ ? . Suppose $t_{i}\left(D, x_{\mathrm{i} i}\right)$ $=0, \quad x^{\mathrm{i} i} 2 D$ for at least two agents, say $i=1,2$. Because $K\left(D, x^{\mathrm{i}}{ }^{i}\right)=$ ? for $i=$ 1,2 , the inductive assumption yields $\varphi\left(D, x^{\mathrm{i} i}\right)=F\left(x^{\mathrm{i}}{ }^{i}\right)$ and, by $(11), \varphi_{\mathrm{i} i}(D, x)=$ $\lambda F_{\mathrm{i} i}(x)$ for $i=1,2$. This implies $\varphi(D, x)=F(x)$ as above.

If $t_{i}\left(D, x_{\mathrm{i} i}\right)=0$ for at most one $i$, then we can assume $t_{j}\left(D, x_{\mathrm{i} j}\right)>0$ for $j=1,2$. We now construct $x_{\alpha}=x+e^{3}$ and $D^{\alpha}$ exactly as above. This is possible because 


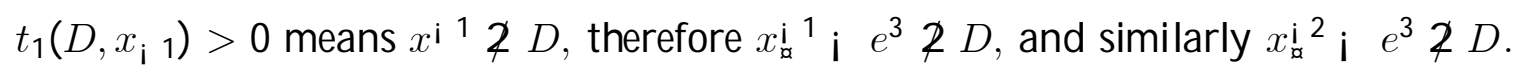
The same argument ends the proof.

Step 5.b. We prove (15) when $\mathbf{j} K(D, x) \mathrm{j}=1$.

In this and the next substep, we use one more piece of notation: given $z, z^{0} 2 \mathbf{R}^{N}$, a $2 \mathbf{R}_{++}^{N}$ and two distinct $i, j 2 N$,

$$
z \gg_{i j}^{a} z^{0}, \quad \frac{z_{i}}{a_{i}} \mathrm{i} \frac{z_{j}}{a_{j}}=\frac{z_{i}^{0}}{a_{i}} \mathrm{i} \frac{z_{j}^{0}}{a_{j}} .
$$

We start by choosing $x$ with $n(x)=3$, say, $N(x)=\mathrm{f} 1,2,3 \mathrm{~g}$ and $D$ such that $K(D, x)=\mathrm{f} 1 \mathrm{~g}$ and $x^{\mathrm{i}}{ }^{1} 2 \mathrm{D}$. We have drawn on Figure 3 the traces of $D$ on the three coordinate hyperplanes. We write $z=\varphi(D, x)$ and $z^{i}=\varphi\left(D, x^{\mathrm{i}}{ }^{i}\right), a_{i}=s_{i}\left(x_{i}\right)$ for all $i$.

Applying (11) to $x, x^{\mathrm{i} 1}$ gives $z_{\mathrm{i}} 1 \mathrm{i} z_{\mathrm{i} 1}^{1}=\mathbf{i} \lambda F_{\mathrm{i}} 1(x)$, hence, by Step 5.a,

$$
\frac{z_{i}}{a_{i}}=\frac{z_{j}}{a_{j}} \text { for all } i, j \in 1 \text {. }
$$

Similarly, (11) applied to $x, x^{\mathrm{i} 2}$ and $x, x^{\mathrm{i} 3}$ gives

$$
\begin{aligned}
& z \gg_{i j}^{a} z^{2} \text { for all } i, j \in 2, \\
& z \gg_{i j}^{a} z^{3} \text { for all } i, j \in 3 .
\end{aligned}
$$

These three properties imply

$$
\frac{z_{1}^{2}}{a_{1}} \mathrm{i} \frac{z_{3}^{2}}{a_{3}}=\frac{z_{1}}{a_{1}} \mathrm{i} \frac{z_{3}}{a_{3}}=\frac{z_{1}}{a_{1}} \mathrm{i} \frac{z_{2}}{a_{2}}=\frac{z_{1}^{3}}{a_{1}} \mathrm{i} \frac{z_{2}^{3}}{a_{2}} .
$$

By Independence of I rrelevant Costs, $z^{2}=\varphi\left(D, x^{\mathrm{i}}{ }^{2}\right)$ only depends upon $x^{\mathrm{i} 2}$ and the trace of $D$ on $\left[0, x^{i}{ }^{2}\right]$. Similarly $z^{3}$ depends only upon $x^{i}{ }^{3}$ and the trace of $D$ on $\left[0, x^{i}{ }^{3}\right]$. Thus the common quantity in (20) only depends on $x_{1}$ and the trace of $D$ on $\left[0, x_{1} e^{1}\right]$, namely $\tau_{1}(D)$. We write this quantity as $\lambda_{1}\left(x_{1}, \tau_{1}(D)\right)$ or $\lambda_{1}$ for simplicity in the rest of this step.

Combining (17), (20), we get

$$
\frac{z_{1}}{a_{1}} \mathrm{i} \frac{z_{i}}{a_{i}}=\lambda_{1} \text { for all } i \in 1
$$

and by (16),

$$
z=H\left(a,\left(\lambda_{1}, 0_{i}\right)\right),
$$

which is precisely (15) for $(D, x)$. 
Next we derive a similar formula for $z^{2}$ and for $w^{1}=\varphi\left(D, x_{1} e^{1}\right)$. A pplying (11) to $x^{\text {i } 2}, x_{1} e^{1}$, we obtain

$$
z^{2} \gg{ }_{i j}^{a^{2}} w^{1} \text { for all } i, j \in 3 \text {, with } a_{2}^{2}=s_{2}(0), a_{i}^{2}=a_{i}, i \in 2 .
$$

Combining (21) and (18), and the fact that $\gg{ }_{i j}^{a}$ is an equivalence relation, we get for all $i, 4$,

$$
\left.w^{1} \gg{ }_{1 i}^{a^{2}} z^{2} \gg_{1 i}^{a} z\right) \quad w^{1} \gg_{1 i}^{a} z, \quad \frac{w_{1}^{1}}{a_{1}} \mathbf{i} \frac{w_{i}^{1}}{a_{i}}=\lambda_{1}
$$

where $a_{i}=s_{i}(0)$. Now $w^{1}$ only depends on $x_{1}$ and $\tau_{1}(D)$, as does $\lambda_{1}$. If we repeat the construction of Step 5.b while exchanging the roles of 2 and 4, i.e., for $x^{0}$ such that $N(x)=\mathrm{f} 1,3,4 \mathrm{~g}$ and $D^{0}$ such that $K\left(D^{0}, x 9=\mathrm{f} 1 \mathrm{~g}\right.$ and $x^{9} 1^{1} 2 D$, we get

$$
\frac{w_{1}^{1}}{a_{1}} \mathbf{i} \frac{w_{2}^{1}}{s_{2}(0)}=\lambda_{1}
$$

Combining these properties of $w^{1}$, including a similar equality where 3 replaces 2 , and (16), we conclude

$$
w^{1}=H\left(a^{1},\left(\lambda_{1}, 0_{i}\right)\right),
$$

where $a_{1}^{1}=s_{1}\left(x_{1}\right)$ and $a_{i}^{1}=s_{i}(0)$ for $i, 2$ : this is precisely (15) for $\left(D, x_{1} e^{1}\right)$. As $\varphi\left(D, x_{1} e^{1}\right)$ only depends on $x_{1}$ and $\tau_{1}(D)$, this establishes (15) for any $(D, x)$ where $n(x)=1$.

Next we compute $z^{2}$. From (18) and (21), we obtain respectively

$$
\begin{aligned}
\frac{z_{1}^{2}}{a_{1}} \mathrm{i} \frac{z_{2}^{2}}{s_{2}(0)} & =\lambda_{1}, \\
\frac{z_{1}^{2}}{a_{1}} \mathrm{i} \frac{z_{i}^{2}}{a_{i}} & =\lambda_{1} \text { for all } i, 3 .
\end{aligned}
$$

Thus (16) implies $z^{2}=H\left(a^{2},\left(\lambda_{1}, 0_{i} 1\right)\right)$. A gain, $\varphi\left(D, x^{i}{ }^{2}\right)$ only depends upon $x^{\mathrm{i}} 2$ and the restriction of $D$ to $\left[0, x^{i}{ }^{2}\right]$. Our choice of $D$ in Step 5.b places no constraint on the restriction of $D$ to $\left[0, x^{\mathrm{i}}{ }^{2}\right.$ ], except $K\left(D, x^{\mathrm{i}}{ }^{2}\right)=\mathrm{f} 1 \mathrm{~g}$. Therefore we have proven (15) for any $(D, x)$ where $n(x)=2$ and $\mathbf{j} K(D, x) \mathbf{j}=1$.

To complete the proof of (15) for any $(D, x)$ such that $n(x)=3$ and $\mathbf{j} K(D, x) \mathbf{j}=1$, it only remains to take care of the case where $x^{\mathrm{i}}{ }^{1} 2 D$ (recall we assumed $x^{\mathrm{i}}{ }^{1} 2 D$ instead at the beginning of Step 5.b). Consider such a con..guration illustrated in Figure 3. We already know that $z^{2}=\varphi\left(D, x^{i^{2}}\right)$ and $z^{3}=\varphi\left(D, x^{i^{3}}\right)$ are given by (15). Applying (11) to $z=\varphi(D, x), z^{2}$ and to $z, z^{3}$ delivers respectively the direction of $z_{\mathrm{i}} 2$ and of $z_{\mathrm{i} 3}$, hence determines $z$ by (16). We omit the details. 
To complete Step 5.b, it remains to establish formula (15) when $\mathbf{j} K(D, x) \mathrm{j}=1$ and $n(x)>3$. We proceed by induction on $n(x)$. The argument is essentially the same as above, once we observe that $K(D, x)=\mathrm{flg}$ implies that for all $i \in 1, x_{i} e^{i} \mathcal{Z} D$, hence $t_{i}\left(D, x_{\mathrm{i} i}\right)=0$.

A ssume the desired conclusion holds whenever $n(x) \cdot t ; 1$ and let $(D, x)$ be such that $K(D, x)=\mathrm{flg}$ and $n(x)=q$. Without loss of generality, assume $N(x)$ contains 2,3. As before (see (21)) we de. ne $a$ by $a_{i}=s_{i}\left(x_{i}\right)$ for all $i$, and $a^{i}$ by $a_{i}^{i}=s_{i}(0)$ and $a_{j}^{i}=a_{j}$ for all $j \boldsymbol{G} i$. Clearly $K\left(D, x^{\mathrm{i}}{ }^{2}\right)=\mathrm{f} 1 \mathrm{~g}$, so the inductive assumption gives

$$
z^{2}=\varphi\left(D, x^{\mathrm{i}}{ }^{2}\right)=H\left(a^{2},\left(\lambda_{1}, 0_{i}\right)\right) .
$$

Applying (11) to $z=\varphi(D, x)$ and $z^{2}$ gives

$$
\frac{z_{1}}{a_{1}} \mathrm{i} \frac{z_{i}}{a_{i}}=\lambda_{1}=\frac{z_{1}^{2}}{a_{1}} \mathrm{i} \frac{z_{i}^{2}}{a_{i}} \text { for all } i, 3 .
$$

The symmetrical argument involving $z$ and $z^{3}$ gives $\frac{z_{1}}{a_{1}} \mathbf{i} \frac{z_{2}}{a_{2}}=\lambda_{1}$ and the proof of Step 5.b is complete.

Step 5.c. We prove (15) by induction on $\mathbf{j} K(D, x) \mathbf{j}$.

$\mathrm{F}$ ix $k, 2$ and assume (15) holds whenever $\mathbf{j} K(D, x) \mathbf{j} \cdot k \mathbf{i} 1$. Consider $(D, x)$ with $\mathbf{j} K(D, x) \mathbf{j}=k$, say, $K(D, x)=\mathrm{f} 1, \ldots, k \mathbf{g}$. De.ne $z=\varphi(D, x), z^{i}=\varphi\left(D, x^{\mathrm{i} i}\right), a$, and $a^{i}$ exactly as in Step 5.b. Clearly $K\left(D, x^{\mathrm{i}}{ }^{i}\right)=\mathrm{f} 1, \ldots, k \operatorname{gnf} i \mathrm{~g}$ for $i \cdot k$, therefore the inductive assumption implies

$$
z^{i}=H\left(a^{i},\left(b_{\mathbf{i}}, 0_{i}\right)\right) \text { for } i=1, \ldots, k,
$$

where $b$ is de. ned by $b_{j}=\lambda_{j}\left(x_{j}, \tau_{j}(D)\right)$ if $j \cdot k$, and $b_{j}=0$ otherwise. Applying (11) successively to $z, z^{2}$ and $z, z^{3}$ gives, for all $i, 3$,

$$
\left.z \gg_{1 i}^{a} z^{2}\right) \quad \frac{z_{1}}{a_{1}} \mathrm{i} \frac{z_{i}}{a_{i}}=b_{1} \mathbf{i} \quad b_{i},
$$

and

$$
\left.z \gg_{12}^{a} z^{3}\right) \frac{z_{1}}{a_{1}} \mathrm{i} \frac{z_{2}}{a_{2}}=b_{1} \mathrm{i} \quad b_{2},
$$

and (16) then yields $z=H(a, b)$, as desired.

Step 6. We construct the functions $\mu_{i}, i 2 N$, and complete the proof.

Rewrite (14) as

$$
H_{i}(a, b)=\frac{\mathrm{p} a_{i}}{{ }_{N} a_{j}}\left(1_{\mathbf{i}}{ }_{N}^{\mathbf{X}} b_{j} a_{j}\right)+a_{i} b_{i} .
$$


De.ne $\mu_{i}, i 2 N$, by

$$
\begin{aligned}
\mu_{i}\left(x_{i}, t_{i}\right)= & s_{i}\left(x_{i}\right) \lambda_{i}\left(x_{i}, t_{i}\right) \text { if } t_{i} \cdot x_{i}, \\
& 0 \text { otherwise. }
\end{aligned}
$$

Recalling our convention $\tau_{i}(D)=+1$ if $D$ does not intersect the $i$-axis, formula (15) can be rewritten as follows. For all $i, x, D$ such that $x 2 D$,

$$
\varphi_{i}(D, x)=\mu_{i}\left(x_{i}, \tau_{i}(D)\right)+F_{i}(x)\left(1_{\mathbf{i}}{ }_{N}^{\mathbf{X}} \mu_{j}\left(x_{j}, \tau_{j}(D)\right)\right) .
$$

This formula coincides with (9) because (8) reduces to $s a^{\mu_{i}}\left(D, x_{i}\right)=\mu_{i}\left(x_{i}, \tau_{i}(D)\right)$ when $D 2 \mathrm{D}$. An additive method is entirely determined by its behavior on such pairs $(D, x)$, thus the proof is complete.

\section{Proof of Theorem $1^{*}$, statement ii)}

We .x throughout an additive method $\varphi$ meeting the four axioms in the statement, denoted for brevity SOL, MON, ZCZD and PCPD. The structure of the proof, and the numbering of the corresponding steps and substeps, are identical to those of the proof of statement i): we compute $\theta(x)$...rst, thus constructing $F$, then $\varphi(D, x)$ by constructing $\mu$ Some of the steps are considerably shorter, however.

Step 1. ZCZD and PCPD imply at once for all $x, D$,

$$
x 2 D) \quad \mathbf{f} \varphi_{i}(D, x)>0, \quad i 2 N(x) \mathbf{g}
$$

and in particular $\theta_{i}(x)>0, \quad x_{i}>0$.

Step 2. We show here a stronger property than (11), namely for all $x, D, i$ such that $x_{i}>0$ and $x_{\mathrm{i} i} \in 0$,

$$
\begin{gathered}
\left.x^{\mathbf{i} i} 2 D\right) \quad \varphi_{\mathrm{i} i}(D, x) \mathbf{i} \quad \varphi_{\mathbf{i} i}\left(D, x^{\mathbf{i} i}\right)=\mathbf{i}_{\lambda} \lambda \theta_{\mathbf{i} i}(x) \text { for some } \lambda>0, \\
\left.x^{\mathrm{i} i} Z D\right) \quad \varphi_{\mathbf{i} i}(D, x)=\lambda \theta_{\mathbf{i} i}(x) \text { for some } \lambda>0 .
\end{gathered}
$$

Note that by taking the dixerence of (22) for $x_{i}$ and $x_{i}^{0}$, we get (11).

To $\beta$ rove (22) and (23) we note that $\varphi_{i}(D, x)<\varphi_{i}\left(D, x^{\mathrm{i}}{ }^{i}\right)$ (by ZCZD and PCPD), hence $N_{N n_{i}} \varphi_{j}(D, x)>\quad N_{N i} \varphi_{j}\left(D, x^{\mathrm{i} i}\right)$. By SOL, $\varphi_{j}(D, x), \varphi_{j}\left(D, x^{\mathrm{i}}{ }^{i}\right)$ for each $j 2 N$ n. Now suppose that for some $j, k 2 N(x)$ ni we have $\varphi_{j}(x)<\varphi_{j}\left(x^{\mathrm{i} i}\right)$ whereas $\varphi_{k}(x)=\varphi_{k}\left(x^{\mathrm{i}}{ }^{i}\right)$. P ick $D^{0}$ containing $x$ but not $x^{0}$ and apply SOL to $\alpha D+D^{0}$ between $x^{\mathrm{i} i}$ and $x$ : 


$$
\mathrm{f} \alpha\left(\varphi_{j}(D, x) \text { i } \varphi_{j}\left(D, x^{\mathrm{i} i}\right)\right)+\varphi_{j}\left(D^{0}, x\right) \mathrm{g} \varphi_{k}\left(D^{0}, x\right), 0,
$$

which contradicts PCPD for $\alpha$ large enough. Thus $\varphi_{j}(D, x) \mathrm{i} \varphi_{j}\left(D, x^{\mathrm{i} i}\right)<0, \quad j 2$ $N(x) \mathrm{n} i$, and for all $\alpha>0$, all $j, k 2 N(x) \mathrm{nf} i \mathrm{~g}$,

$$
\mathrm{f} \alpha\left(\varphi_{j}(D, x) \text { i } \varphi_{j}\left(D, x^{\mathrm{i} i}\right)\right)+\varphi_{j}\left(D^{0}, x\right) \text { gf } \alpha\left(\varphi_{k}(D, x) \text { i } \varphi_{k}\left(D, x^{\mathrm{i} i}\right)\right)+\varphi_{k}\left(D^{0}, x\right) \mathrm{g}, 0,
$$

implying $\varphi_{\mathrm{i} i}(D, x) \mathbf{\mathrm { i }} \varphi_{\mathrm{i} i}\left(D, x^{\mathrm{i}}{ }^{i}\right)=\mathbf{i} \nu \varphi_{\mathrm{i} i}\left(D^{0}, x\right)$ for some $\nu>0$. If we choose $D^{0}$ so that $x 2 \partial D^{0}$ we get (22), and (23) follows.

Step 3. The direction of the vector $\theta_{i} i(x)$ is independent of $x_{i} 2 \mathrm{NnfOg}$, when $x_{\mathrm{i} i} \in 0$.

Indeed, pick $x_{i}, x_{i}^{0}$ such that $0<x_{i}<x_{i}^{0}$, and $D$ such that $\left(x_{i}, x_{\mathbf{i} i}\right) 2 \partial D$. By (23), $\varphi_{\mathrm{i} i}\left(D,\left(x_{i}^{0}, x_{\mathbf{i} i}\right)\right)=\lambda \theta_{\mathbf{i} i}\left(\left(x_{i}^{0}, x_{\mathbf{i} i}\right)\right)$ for some $\lambda>0$ and by $(11) \varphi_{\mathbf{i} i}\left(D,\left(x_{i}^{0}, x_{\mathbf{i} i}\right)\right)$ i $\varphi_{\mathbf{i} i}\left(D,\left(x_{i}, x_{\mathbf{i} i}\right)\right)=\varphi_{\mathbf{i} i}\left(D,\left(x_{i}^{0}, x_{\mathbf{i} i}\right)\right) \mathbf{i} \quad \theta_{\mathbf{i} i}\left(\left(x_{i}, x_{\mathbf{i} i}\right)\right)=\mathbf{i} \mu \theta_{\mathbf{i} i}(x)$ for some $\mu, 0$.

Step 4. We construct the nondecreasing functions $s_{i}$ such that $s_{i}(0)=0<s_{i}(1)$ and $\theta(x)=F(x)$ for all $x$ such that $n(x), 3$.

Setting $N_{0}=N(x)$ and $\theta_{0}(x)$ to be the projection of $\theta(x)$ on $\mathbf{R}^{N_{0}}$, we note that $\theta_{0}(x)$ is strictly positive and in $\phi\left(N_{0}\right)$. By Step $3, \frac{\theta_{0 i}}{\theta_{0 j}}$ depends on $x_{i}, x_{j}$ only, and a standard argument gives then the functions $s_{i}$ on Nnfog such that $\theta_{0}(x)=F(x)$ for all $x$ such that $N(x)=N_{0}$. The argument is essentially the same as in Step 4 of the previous proof, except that we only need $N_{0}$ to contain 3 or more agents. This is why we only need to assume $n, 3$ in statement ii) of Theorem $1^{*}$.

In view of Step1, it is now routine to show that the functions $s_{i}$ do not depend on $N_{0}$, and to extend the equality $\theta(x)=F(x)$ to all coordinates by setting $s_{i}(0)=0$.

Step 5. We show, as in the previous proof, the existence of functions $\lambda_{i}$ allowing the representation of $\varphi(D, x)$ by (15).

Step 5.a. For any $(D, x)$ with $x 2 D$ and $K(D, x)=?, \varphi(D, x)=F(x)$.

P rove this ..rst for $x$ such that $N(x)=\mathrm{f} 1,2 \mathrm{~g}$. Set $x_{\mathrm{\alpha}}=x+e^{3}$, pick $D^{\mathrm{a}}$ such that $D^{\alpha}=D$ on $[0, x]$ and $x_{\alpha}^{\mathrm{i}}{ }^{1}, x_{\alpha}^{\mathrm{i}}{ }^{2} 2 D^{\alpha}$. By (23) $\varphi_{\mathrm{i} i}\left(D^{\alpha}, x_{\alpha}\right)$ is borne by $F_{\mathrm{i} i}\left(x_{\alpha}\right)$ for $i=1$, 2. In view of Step 4 this implies $\varphi\left(D^{\mathfrak{a}}, x_{\mathrm{a}}\right)=F\left(x_{\mathrm{a}}\right)$. Invoke next (22): $\varphi_{\mathrm{i} 3}\left(D^{\mathfrak{\alpha}}, x_{\mathfrak{\alpha}}\right) \mathrm{i} \varphi_{\mathrm{i} 3}\left(D^{\alpha}, x\right)=\mathrm{i} \lambda F_{\mathrm{i} 3}\left(x_{\mathfrak{\alpha}}\right)$. Therefore $\frac{s_{1}\left(x_{1}\right)}{s_{2}\left(x_{2}\right)}=\frac{\varphi_{1}}{\varphi_{2}}\left(D_{\mathfrak{\alpha}}, x\right)=\frac{\varphi_{1}}{\varphi_{2}}(D, x)$, where the latter equality follows from Independence of Irrel evant Costs.

The ascending induction on $n(x)$ is now immediate by (22) and Step 1.

Step 5.b. We assume now $\mathrm{j} K(D, x) \mathrm{j}=1$, and of course $x 2 D$. If $n(x)=1$ there is nothing to prove (by ZC ZD), contrary to the previous proof. If $n(x)=2$, we simply copy Step 5.b to de. ne the functions $\lambda_{i}$ and compute $z, z^{2}, z^{3}$. By Step 1 we 
need not worry about whether or not $x^{\mathrm{i}}{ }^{1} 2 D$. The ascending induction on $n(x)$ is unchanged.

Step 5.c. The ascending induction on $\mathbf{j} K(D, x) \mathbf{j}$ is unchanged.

Step 6. is unchanged.

8.3. Proof of Theorem 1. The "if" part of statements i) and ii) is clear. To prove the "only if" part in either statement, we assume that a method given by (9) satis.. es Strong Ranking, and prove that $\mu_{i}$, hence $s a^{\mu_{i}}$ is identically zero for all $i$.

We check ..rst $s_{i}=s_{j}$ for all $i, j$. Pick $x=t e^{N}, t, 1$, and $C, C(z)=1$ if $n(z), 2, C(z)=0$ otherwise. Then $\varphi(C, x)=F(x)$, so Strong Ranking implies $s_{i}(t)=s_{j}(t)$ for $t, 1$. Under Zero Cost for Zero Demand, this is enough. On the other hand if $n$, 4, we choose $x^{0}=t e^{N \text { nf } 1,2 \mathrm{~g}}$ and the same cost function $C$ to get $s_{1}(0)=s_{2}(0)$, and we are done.

A ssume now there is an agent $i$ and $x_{1}, t_{1}$ such that $\mu_{1}\left(x_{1}, t_{1}\right) \in 0$. Choose $C=$ $\delta^{\left(t_{1}, 0_{i}\right)}$ as in (28) so that $s a^{\mu_{i}}\left(C, x_{i}\right)=0$ for $i, 2, s a^{\mu_{1}}\left(C, x_{1}\right)=\mu_{1}\left(x_{1}, t_{1}\right)$. Then for $i, 2$,

$$
\varphi_{1}\left(C, x_{1} e^{N}\right)=\mu_{1}+\frac{1}{n}\left(1 \text { i } \mu_{1}\right) \Leftrightarrow \frac{1}{n}\left(1 \text { i } \mu_{1}\right)=\varphi_{i}\left(C, x_{1} e^{N}\right),
$$

contradicting Strong R anking.

8.4. Proof of Theorem 2. Step 1. i) implies ii) and iii). Let $\varphi$ be a ..xed-łow method. It is easy to check that $\varphi$ satis. es A dditivity, Separability and M onotonicity; we prove here that it also meets Submodular Solidarity and Supermodular Solidarity. Let $f(., \bar{x})$ be the ..xed-łow associated with $\varphi$ and write $f(., x)=p_{x} f(., \bar{x})$ for all $x 2[0, \bar{x}]$. Observe that $p_{x} f\left(., x+e^{i}\right)=f(., x)$ for all $i$, all $x 2\left[\bar{x} \mathbf{i} e^{i}\right]$. We ..x $i, j$, $i \boldsymbol{6} j, x \mathbf{2}\left[\bar{x} \mathbf{i} e^{i}\right]$, and $z 2\left[x \mathbf{i} e^{j}\right]$.

First we compare $f_{j}(z, x)$ and $f_{j}\left(z, x+e^{i}\right)$. If $z_{i}<x_{i}$, then $K$ contains neither $i$ nor $j$, hence $\left(x+e^{i}\right)_{K}=x_{K}$, implying $f_{j}(z, x)=f_{j}\left(z, x+e^{i}\right)$. If $z_{i}=x_{i}$, then $K$ contains $i$ but not $j$, hence $\left[x_{K},\left(x+e^{i}\right)_{K}\right]=\mathbf{f} x_{K}, x_{K}+e_{K}^{i} \mathbf{g}$ so that $f_{j}(z, x)=$ $f_{j}\left(z, x+e^{i}\right)+f_{j}\left(z+e^{i}, x+e^{i}\right)$.

Now we compute

$$
=\begin{gathered}
\varphi_{j}\left(C, x+e^{i}\right) \mathbf{i} \varphi_{j}(C, x) \\
\quad\left(\partial_{j} C\left(z+e^{i}\right) f_{j}\left(z+e^{i}, x+e^{i}\right)+\right. \\
z 2[0, x]: z_{i}=x_{i}, z_{j}<x_{j} \\
\left.\partial_{j} C(z)\left(f_{j}\left(z, x+e^{i}\right) \mathbf{i} f(z, x)\right)\right)
\end{gathered}
$$


implying Submodular Solidarity and Supermodular Solidarity at once.

Step 2. ii) implies i). Fix a method $\varphi$ satisfying Additivity, Separability, M onotonicity, and Submodular Solidarity. Let $f$ be its $\neq$ ow representation as in (5). We show $f(., x)=p_{x} f(., \bar{x})$ for any $x 2[0, \bar{x}]$.

Step 2.a. For all $i 2 N, x \mathbf{2}\left[0, \bar{x} \mathbf{i} e^{i}\right]$, and $z 2\left[0, x \mathbf{i} e^{i}\right]$,

$$
f_{i}(z, x)=f_{i}\left(z, x+e^{i}\right) .
$$

This is a well-known consequence of Monotonicity: see Moulin (1995) or Sprumont (2000) for a proof. Equation (24) does not imply the property $f_{j}(z, x)=f_{j}\left(z, x+e^{i}\right)$ for $j \in i$ (except when $n=2$, where it follows from $\neq$ ow conservation); that property is derived in the next step using Submodular Solidarity.

Step 2.b. For all $i 2 N, x \mathbf{2}\left[0, \bar{x} \mathbf{i} e^{i}\right]$, and $z 2\left[0, x \mathbf{i} e^{i}\right]$,

$$
f(z, x)=f\left(z, x+e^{i}\right) .
$$

The proof of (25) is by induction on $z(N)$, the sum of the coordinates of $z$. Let $z=0$. By Step 2.a, $f_{i}(0, x)=f_{i}\left(0, x+e^{i}\right)$. If (25) fails, as total out $\neq$ ow from 0 is 1 under $f(., x)$ and $f\left(., x+e^{i}\right)$, there exist $j, k 2 N$ ni such that $f_{j}(0, x)<f_{j}\left(0, x+e^{i}\right)$ and $f_{k}(0, x)>f_{k}\left(0, x+e^{i}\right)$. Consider the cost function

$$
\begin{aligned}
\delta_{0}(w)= & 0 \text { if } w=0, \\
& 1 \text { otherwise. }
\end{aligned}
$$

Note that $\delta_{0} 2 C_{\text {sub. }}$ But $\varphi_{j}\left(\delta_{0}, x\right)=f_{j}(0, x)<f_{j}\left(0, x+e^{i}\right)=\varphi_{j}\left(\delta_{0}, x+e^{i}\right)$ and $\varphi_{k}\left(\delta_{0}, x\right)=f_{k}(0, x)>f_{k}\left(0, x+e^{i}\right)=\varphi_{k}\left(\delta_{0}, x+e^{i}\right)$, contradicting Submodular Solidarity.

Next, ..x $k>0$ and assume that (25) is true for all $z 2\left[0, x \mathbf{i} e^{i}\right.$ [ such that $z(N) \cdot k \mathbf{i} 1 . \mathrm{Fix} z 2\left[0, x \mathbf{i} e^{i}\right.$ [ such that $z(N)=k$. By the induction hypothesis $f_{j}\left(z \mathbf{i} e^{j}, x\right)=f_{j}\left(z \mathbf{i} \quad e^{j}, x+e^{i}\right)$ for all $j 2 N$ such that $z_{j}>0$, hence the total incoming fow at $z$ is the same under $f(., x)$ and $f\left(., x+e^{i}\right)$ :

$$
\underset{j 2 N(z)}{\mathbf{X}} f_{j}\left(z \mathbf{i} e^{j}, x\right)={ }_{j 2 N(z)}^{\mathbf{X}} f_{j}\left(z \mathbf{i} e^{j}, x+e^{i}\right) .
$$

Conservation of łows and (24) imply now ${ }^{\mathrm{P}}{ }_{j 2 \mathrm{Nni}} f_{j}(z, x)={ }_{j 2 \mathrm{Nni}} f_{j}\left(z, x+e^{i}\right)$. If $z_{j}<x_{j}$ for at most one $j 2 \mathrm{Nn}$, we are done Otherwise suppose, by contradiction, that $f(z, x) \in f\left(z, x+e^{i}\right)$ : there exist $j, k 2 N$ ni such that $f_{j}(z, x)<f_{j}\left(z, x+e^{i}\right)$ and $f_{k}(z, x)>f_{k}\left(z, x+e^{i}\right)$. Consider the cost function

$$
\begin{aligned}
\delta_{z}(w)= & 0 \text { if } w \cdot z, \\
& 1 \text { otherwise. }
\end{aligned}
$$


A gain, $\delta_{z} 2 \mathrm{C}_{\text {sub }}$. Compute

$$
\begin{aligned}
& \varphi_{j}\left(\delta_{\tilde{\mathbf{X}}}, x+e^{i}\right) \mathbf{i} \varphi_{j}\left(\delta_{z}, x\right) . \\
= & \quad\left(f_{j}\left(\left(z_{j}, w_{N \mathrm{n} j}\right), x+e^{i}\right) \mathbf{i} f_{j}\left(\left(z_{j}, w_{N \mathrm{n} j}\right), x\right)\right) \\
= & w_{N \mathrm{n} j} 2\left[0, z_{\left.N \mathrm{n}_{j}\right]}\left(z, x+e^{i}\right) \mathbf{i} f_{j}(z, x)>0\right.
\end{aligned}
$$

and, symmetrically, $\varphi_{k}\left(\delta_{z}, x+e^{i}\right) \mathrm{i} \quad \varphi_{k}\left(\delta_{z}, x\right)=f_{k}\left(z, x+e^{i}\right) \mathrm{i} f_{k}(z, x)<0$, contradicting Submodular Solidarity.

Step 2.c. For all $i 2 N, x 2\left[0, \bar{x} \mathbf{i} e^{i}\right]$, and $z 2[0, x[$,

$$
f(z, x)=p_{x} f\left(z, x+e^{i}\right) .
$$

Since $p_{x} p_{x} f(., \bar{x})=p_{x^{0}} f(., \bar{x})$ for $x^{0} \cdot x \cdot \bar{x}$, this will complete the proof of Step 2.

By Step 2.b, (27) is true for $z 2$ [0, $x$ i $e^{i}$ [ as for such a $z$ we have $i 2 K$ and $x_{K}=\left(x+e^{i}\right)_{K}$. It remains to extend it to all $z 2\left[x_{i} e^{i}, x\right.$. Consider ..rst $z=x_{i} e^{i}$ and suppose, by way of contradiction, that $f\left(x_{i} e^{i}, x\right) \boldsymbol{\sigma} p_{x} f\left(x_{i} e^{i}, x+e^{i}\right)$ : there exist $j, k 2 N$ ni such that $f_{j}\left(x_{i} e^{i}, x\right)>f_{j}\left(x_{i} e^{i}, x+e^{i}\right)+f_{j}\left(\left(x_{i}+1\right) e^{i}, x+e^{i}\right)$ and $f_{k}\left(x_{i} e^{i}, x\right)<$ $f_{k}\left(x_{i} e^{i}, x+e^{i}\right)+f_{k}\left(\left(x_{i}+1\right) e^{i}, x+e^{i}\right)$. Recalling Step 2.b, this yields a violation of

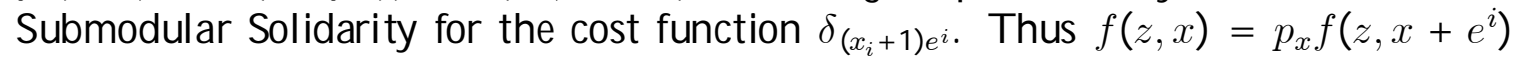
for $z=x_{i} e^{i}$. An induction argument mimicking that in Step 2.b completes Step 2.c, and the proof that $\varphi$ is a ..xed-łow method.

Step 3. iii) implies i). The argument in Step 2 is easily adapted. T he submodular cost functions $\delta_{z}$ are merely replaced with the supermodular functions $\delta^{z}$ de..ned by

$$
\begin{aligned}
\delta^{z}(w)= & 1 \text { if } w, z, \\
& 0 \text { otherwise, }
\end{aligned}
$$

and the induction argument is carried on $x(N) \mathbf{i} z(N)$ rather than on $z(N)$.

8.5. Proof of Proposition 5. Step 1 of the proof of Theorem 2 shows that the ..xed- $\ddagger$ ow methods meet Positive Externalities and Negative Externalities.

Next, we show that the Aumann-Shapley method (Example 1) also does. To shorten notation, we denote $\varphi$ the A umann-Shapley method, and $f$ its $\ddagger$ ow representation. Fix a demand pro..le $x$, two arbitrary agents 1 and 2 , and $z_{\mathrm{i}} 12\left[0_{\mathrm{i}}, x_{\mathrm{i}}[\right.$. For $z_{1}=0, \ldots, x_{1}+1$, we write $f\left(z_{1}, x\right)$ instead of $f\left(\left(z_{1}, z_{i}\right), x\right)$ and $f\left(z_{1}, x+e^{1}\right)$ instead of $f\left(\left(z_{1}, z_{\mathrm{i}} 1\right), x+e^{1}\right)$. We claim that

$$
\mathrm{X}_{z_{1}=0}^{\chi_{1}} f_{2}\left(z_{1}, x\right)=\underbrace{\chi^{+1}}_{z_{1}=0} f_{2}\left(z_{1}, x+e^{1}\right)
$$


and, for $k=0, \ldots, x_{1}$ i 1 ,

$$
\mathrm{X}_{z_{1}=0}^{k} f_{2}\left(z_{1}, x\right), \mathrm{X}_{z_{1}=0}^{\mathrm{X}^{k}} f_{2}\left(z_{1}, x+e^{1}\right) .
$$

The claim implies that the sequence $\left(f_{2}\left(z_{1}, x\right) ; z_{1}=0, . ., x_{1}\right)$, augmented by 0 as the last term, stochastically dominates $\left(f_{2}\left(z_{1}, x+e^{1}\right) ; z_{1}=0, . ., x_{1}+1\right)$. Using (5), this implies $\varphi_{2}(C, x), \varphi_{2}\left(C, x+e^{1}\right)$ if $C$ is submodular and the reverse weak inequality holds if $C$ is supermodular.

E quation (29) follows immediately from the fact that the A umann-Shapley method satis..es Strong Dummy (see comment 2 after Theorem 2). We omit the easy proof.

To prove (30), recall the notation and formula in Example 1 and de.ne $\beta_{k}=$ $\alpha\left(\left(k, z_{\mathrm{i} 1}\right)\right), \gamma_{k}=\alpha\left(x \mathbf{i} \quad\left(k, z_{\mathrm{i} 1}\right) \mathrm{i} e^{2}\right)$ for $k=0, \ldots, x_{1} \mathrm{i} \quad 1$, and $\gamma_{\mathrm{i} 1}=\alpha\left(x \mathbf{i} \quad\left(0, z_{\mathrm{i} 1}\right)+\right.$ $\left.e^{1} \mathrm{i} e^{2}\right)$. N ote that

$$
\begin{aligned}
\frac{\alpha\left(x+e^{1}\right)}{\alpha(x)} & =\frac{x(N)+1}{x_{1}+1}, \\
\beta_{k} & =\frac{\mathbb{Q}_{t=1}(z(N \mathrm{n} 1)+t)}{k !} \beta_{0}, \\
\gamma_{k} & =\frac{\mathbb{Q}\left(x_{1} \mathrm{i} t+1\right)}{\mathbb{Q}(x(N) \mathrm{i} z(N \mathrm{n} 1) \mathrm{i} t)} \gamma_{0} .
\end{aligned}
$$

Writing $a_{k}=z(N \mathrm{n} 1)+k$ and $b_{k}=x(N) \mathbf{i} \quad z(N \mathrm{n} 1) \mathrm{i} \quad k,(30)$ reads

$$
\begin{aligned}
& \frac{x(N)+1}{x_{1}+1}\left(1+\frac{a_{1} x_{1}}{b_{1}}+\frac{a_{1} a_{2} x_{1}\left(x_{1} \mathrm{i} 1\right)}{2 b_{1} b_{2}}+\ldots+\frac{a_{1} \ldots a_{k} x_{1} \ldots\left(x_{1} \mathrm{i} k+1\right)}{k ! b_{1} \ldots b_{k}}\right) \\
& \frac{b_{0}}{x_{1}+1}+a_{1}+\frac{a_{1} a_{2} x_{1}}{2 b_{1}}+\ldots+\frac{a_{1} \ldots a_{k} x_{1} \ldots\left(x_{1} \mathrm{i} k+2\right)}{k ! b_{1} \ldots b_{k \mathrm{i}} 1}
\end{aligned}
$$

Using $x(N)+1=a_{1}+b_{0}$, subtract $\frac{b_{0}}{x_{1}+1}$ from both sides of this inequality and divide each side by $a_{1}$ to obtain the equivalent inequality

$$
\begin{aligned}
& \frac{1}{x_{1}+1}+\frac{x(N)+1}{x_{1}+1}\left(\frac{x_{1}}{b_{1}}+\frac{a_{2} x_{1}\left(x_{1} \mathrm{i} 1\right)}{2 b_{1} b_{2}}+\ldots+\frac{a_{2} \ldots a_{k} x_{1} \ldots\left(x_{1} \mathrm{i} k+1\right)}{k ! b_{1} \ldots b_{k}}\right) \\
& 1+\frac{a_{2} x_{1}}{2 b_{1}}+\ldots+\frac{a_{2} \ldots a_{k} x_{1} \ldots\left(x_{1} \mathrm{i} k+2\right)}{k ! b_{1} \ldots b_{k \mathrm{i}} 1}
\end{aligned}
$$


Subtract $\frac{1}{x_{1}+1}$ from both sides and divide by $x_{1}$ to obtain

$$
\begin{aligned}
& \frac{x(N)+1}{x_{1}+1}\left(\frac{1}{b_{1}}+\frac{a_{2}\left(x_{1} \mathrm{i} 1\right)}{2 b_{1} b_{2}}+\ldots+\frac{a_{2} \ldots a_{k}\left(x_{1} \mathrm{i} 1\right) \ldots\left(x_{1} \mathrm{i} k+1\right)}{2 \ldots k b_{1} \ldots b_{k}}\right) \\
& \frac{1}{x_{1}+1}+\frac{a_{2}}{2 b_{1}}+\ldots+\frac{a_{2} \ldots a_{k}\left(x_{1} \mathrm{i} 1\right) \ldots\left(x_{1} \mathrm{i} k+2\right)}{2 \ldots k b_{1} \ldots b_{k_{\mathrm{i}}} 1}
\end{aligned}
$$

in which $a_{1}$ and $b_{0}$ no longer appear.

Next, using $x(N)+1=a_{2}+b_{1}$, a similar two-step argument reduces that inequality to the equival ent

$$
\begin{aligned}
& \frac{x(N)+1}{x_{1}+1}\left(\frac{1}{b_{2}}+\frac{a_{3}\left(x_{1} \mathrm{i} 2\right)}{3 b_{2} b_{3}}+\ldots+\frac{a_{3} \ldots a_{k}\left(x_{1} \mathrm{i} 2\right) \ldots\left(x_{1} \mathrm{i} k+1\right)}{3 \ldots k b_{2} \ldots b_{k}}\right) \\
& \frac{1}{x_{1}+1}+\frac{a_{3}}{3 b_{2}}+\ldots+\frac{a_{3} \ldots a_{k}\left(x_{1} \mathrm{i} 2\right) \ldots\left(x_{1} \mathrm{i} k+2\right)}{3 \ldots k b_{2} \ldots b_{k_{\mathrm{i}}}},
\end{aligned}
$$

where $a_{2}$ and $b_{1}$ no longer appear.

Repeating this procedure $k \mathbf{i} 1$ times establishes that the original inequality(30) is equivalent to

$$
\frac{x(N)+1}{x_{1}+1}\left(\frac{1}{b_{k \mathrm{i}} 1}+\frac{a_{k}\left(x_{1} \mathrm{i} k+1\right)}{k b_{k_{\mathrm{i}} 1} b_{k}}\right), \frac{1}{x_{1}+1}+\frac{a_{k}}{k b_{k_{\mathrm{i}} 1}} .
$$

Using $x(N)+1=a_{k}+b_{k_{i} 1}$, subtracting $\frac{1}{x_{1}+1}$ from both sides and multiplying by $\frac{b_{k i 1} 1}{a_{k}}$, we get $\frac{1}{x_{1}+1}+\frac{(x(N)+1)\left(x_{1 i} k+1\right)}{\left(x_{1}+1\right) k b_{k}}, \frac{1}{k}$, which reduces to $x(N)+1, b_{k}$.

8.6. Statement and proof of Lemma 3. We work out the restrictions imposed by Strong Ranking and Weak Separability on formula (7). For any $x 2 \mathrm{~N}^{N}$, let $\$(x)=\mathbf{f} y \mathbf{2} \$$ jfor all $\left.i, j 2 N, x_{i} \cdot x_{j}\right) \quad y_{i} \cdot y_{j}$ g. Write $\mathbf{f} x_{i} \mathbf{j} i 2 N(x) \mathbf{g}=$ $\mathbf{f} \xi_{1}, \ldots, \xi_{K} \mathbf{g}$, where $0<\xi_{1}<\ldots<\xi_{K}$, and set $\xi_{0}=0$. For $k=1, \ldots, K$, let $N_{k}=$ f $i 2 N \mathbf{j} x_{i}, \xi_{k}$ g. Using this notation, the extreme points of $\phi(x)$ are the vectors $b^{k}=\frac{1}{\mathrm{j} N_{k} \mathrm{j}} e^{N_{k}}, k=1, \ldots, K$. We sometimes write $K(x)$ to emphasize that $K$ depends upon $x$.

Lemma 3. An additive cost-sharing method $\varphi$ satis..es Strong Ranking and W eak Separability if and only if it possesses a shared-łow representation $(f, r)$ such that, for all $x \mathbf{2} \mathbf{N}^{N}, z 2[0, x[, j 2 \mathrm{~N}$, and $k 2 \mathrm{f} 1, \ldots, K(x) \mathrm{g}$,

$$
\left.\mathbf{f} \xi_{k i \mathbf{1}} \cdot z_{j}<\xi_{k} \mathbf{g}\right) \quad \mathbf{f} r(z, j, x)=b^{k} \mathbf{g} .
$$


Proof. Step 1. We show that an additive cost-sharing method $\varphi$ satis..es Strong Ranking if and only if it possesses a shared-łow representation $(f, r)$ such that, for all $x 2 N^{N}, z 2[0, x[$, and $j 2 N, r(z, j, x) 2 \pitchfork(x)$.

The "if" statement is clear from the representation in (7). Conversely, assume $\varphi$ meets A dditivity and Strong R anking, and ..X $x 2 \mathrm{~N}^{N}$ such that $x_{1} \cdot \ldots \cdot x_{n}$. De..ne the linear isomorphism $h: \mathbf{R}^{N} ! \mathbf{R}^{N}$ by

$$
h(y)=\left(n y_{1},(n \mathbf{i} 1)\left(y_{2} \mathbf{i} y_{1}\right), \ldots, 2\left(y_{n \mathbf{i}} 1 \mathbf{i} \quad y_{n \mathbf{i}} 2\right), y_{n} \mathbf{i} y_{n \mathbf{i}}\right)
$$

and observe that it maps $\phi(x)$ into a face - $(x)$ of $\phi$. For instance, if $x_{1}, \ldots, x_{n}$ are all distinct, $h$ is an isomorphism from $\$(x)$ into $\$$; if $N=\mathrm{f} 1,2,3,4,5 \mathrm{~g}$ and $x_{1}=x_{2}<$ $x_{3}<x_{4}=x_{5}$, then $-(x)$ is the face $y_{2}=y_{5}=0$.

De.ne $\psi(C, x)=h(\varphi(C, x))$ for all $C 2 \mathrm{C}$. By Strong R anking, $\varphi(C, x)$ is borne by a vector in $\phi(x)$, hence $\psi(C, x), 0$. Therefore $\psi(., x)$ is a bona ..de additive cost-sharing method at $x$. Thus it has a shared- $\ddagger$ ow representation

$$
\psi(C, x)={ }_{z 2[0, x[j 2 N}^{\mathbf{X}} \partial_{j} C(z) g_{j}(z, x) r(z, j, x)
$$

for all $C 2 \mathrm{C}$.

If $y_{i}=0$ for all $y 2-(x)$, then $\psi_{i}(C, x)=0$ for all $C 2 \mathrm{C}$, implying that $r_{i}(z, j, x)=0$ for all $z 2[0, x$ [ and $j 2 N$. Thus $r(z, j, x) 2-(x)$ for all $z$ and $j$. A pplying $h^{i}$ to both sides of (32), we obtain

$$
\varphi(C, x)=\underbrace{\mathbf{X}}_{z 2[0, x[j 2 N} \partial_{j} C(z) g_{j}(z, x) h^{i 1}(r(z, j, x))
$$

for all $C 2 \mathrm{C}$. Since $h^{\mathrm{i}}{ }^{1}(r(z, j, x)) 2 ф(x)$, we are done.

Step 2. We complete the proof. Let $\varphi$ be a cost-sharing method satisfying Additivity, Strong Ranking, and Weak Separability, and ..x $x 2 \mathrm{~N}^{N}$. For a $2 \mathrm{Nnf} 0 \mathrm{~g}$ and $i 2 N$, consider the function $\delta^{a e^{i}}$ de. ned in $(28): \delta^{a e^{i}}(z)=1$ if $z_{i}, a$ and $\delta^{a e^{i}}(z)=0$ otherwise. By Weak Separability, $\varphi\left({ }_{i 2 N} \delta^{a e^{i}}, x\right)=e^{\mathrm{f} i 2 N \mathrm{Nj} x_{i}, a \mathrm{~g}}$ for all a $2 \mathrm{Nnf} 0 \mathrm{~g}$. Equivalently,

$$
\text { for all } k 2 \mathrm{f} 1, \ldots, K \mathrm{~g} \text { and } \xi_{k_{\mathrm{i} 1}}<a \cdot \xi_{k},{ }_{i 2 N_{k}}^{\mathrm{X}} \varphi\left(\delta^{a e^{i}}, x\right)=e^{N_{k}} .
$$

By Strong Ranking $\varphi\left(\delta^{a e^{i}}, x\right) 2 \$(x)$ for all $i$. By (33) and the fact that $b^{k}$ is an extreme point of $\phi(x), \varphi\left(\delta^{a e^{i}}, x\right)=b_{\mathrm{P}}^{k}$ whenever $\xi_{k_{\mathrm{i}} 1}<a \cdot \xi_{k}$ and $i 2 N_{k}$. If $\varphi$ is represented by $(f, r), \varphi\left(\delta^{a e^{i}}, x\right)={ }_{z: z_{i}=a_{\mathrm{i}} 1} f_{i}(z, x) r(z, i, x)$, therefore the same 
extremality argument yields $r(z, i, x)=b^{k}$ for all $z 2$ [0, $x$ [ such that $z_{i}=a \mathrm{i} 1$ and $f_{i}(z, x)>0$. Note that $z_{i}$ varies from $\xi_{k_{\mathrm{i}} 1}$ to $\xi_{k} \mathrm{i}$ l. If $f_{i}(z, x)=0$, the choice of $r(z, i, x)$ is irrelevant and we may set $r(z, i, x)=b^{k}$ in that case too. This proves the "only if" part of the lemma. The proof of the çnverse statement is similar because every symmetric separable cost function $C(z)={ }_{i 2 N} c\left(z_{i}\right)$ may be written as a linear combination of cost functions $\delta^{a e^{i}}$.

It is interesting to compare the shared- $\ddagger$ ow representation in Lemma 3 with that of separable methods given in formula (5) in Section 4. In both cases the share system $r$ in the general formula (7) is entirely determined and the fow $f$ is arbitrary. Thus the two families of methods -separable on one hand, weakly separable and meeting Strong Ranking on the other hand- are comparably rich. In the former case, however, every fow $f$ yields a dixerent method, whereas in the latter case dixerent $\ddagger$ ows may lead to the same method. Consider for instance the cross-subsidizing serial method $\varphi^{c s}$ (Example 5) and ... $x \times 2 \mathrm{~N}_{\mathrm{x}}^{N}$. Every $\neq$ ow $f(., x)$ that goes entirely through $x^{1}, x^{2}, . ., x^{n}$, together with the sharing rule $r(., ., x)$ de..ned by formula (31), determines a valid representation of $\varphi^{c s}(., x)$.

8.7. Proof of Proposition 7. A ssume $n, 3$ and let $\varphi$ be a cost-sharing method satisfying all four stated axioms. By Lemma 3, $\varphi$ has a shared- $¥$ ow representation $(f, r)$ such that for all $x 2 \mathbf{N}^{N}, z 2[0, x[, j 2 N$, and $k 2 \mathrm{f} 1, \ldots, K(x) \mathrm{g}$, equation (31) holds. In particular, this implies Zero Cost for Zero Demand because $x_{i}=0$ implies $b_{i}^{k}=0$ for all $k=1, \ldots, K(x)$. As a consequence, any contradiction derived for $n=3$ extends to more agents by considering demand pro..les where all agents but three demand zero.

Fix thus $n=3$ and let $x$ be such that $2 \cdot x_{1}<x_{2}<x_{3}$. Write $f$ for $f(., x)$. As already noted in the proof of Proposition 1, Strong Group M onotonicity is equivalent to the property that for all $D 2 \mathrm{D}$, all distinct agents $i, j 2 N$, and all $x 2 \mathbf{N}^{N}$,

$$
D(x)=1) \quad \varphi_{j}\left(D, x+e^{i}\right) \cdot \varphi_{j}(D, x) .
$$

In the foll owing argument, we write $D \quad D^{0}$ for the supremum of two cost functions $D, D^{0} 2 \mathrm{D}$, that is, $\left(D_{-} D^{\mathrm{gi}^{1}}=D^{\mathrm{i}}{ }^{1}(1)\left[D^{\mathrm{q}} \mathrm{l}(1)\right.\right.$. Choose $e_{1} 2\left[0, x_{1}\right.$ [ and set

$$
D=\delta^{\left(0,0, x_{3}\right)}-\delta^{\left(\mathbb{\otimes}_{1}+1,0,0\right)},
$$

where $\delta^{z}$ is the cost function de..ned in (28). By (31) and Independence of Irrel evant Costs, $\varphi\left(D,\left(e_{1}, x_{2}, x_{3}\right)\right)=e^{3}$ because $D$ coincides with $\delta^{\left(0,0, x_{3}\right)}$ on $\left[0,\left(\Theta_{1}, x_{2}, x_{3}\right)\right]$. By (34), $\varphi_{2}(D, x) \cdot \varphi_{2}\left(D,\left(e_{1}, x_{2}, x_{3}\right)\right)$, hence $\varphi_{2}(D, x)=0$. By (31) again, this gives $f_{1}\left(z \mathrm{i} e^{1}\right)=0$ for all $z$ such that $z_{1}=e_{1}+1$ and $z_{3}<x_{3}$. Since this is true for all $\mathbb{e}_{1} 2\left[0, x_{1}\right.$ [, the entire fow must be contained in $A=\mathbf{f} z 2\left[0, x\left[\mathrm{j} z_{1}=0\right.\right.$ or $z_{3}=x_{3} \mathrm{~g}$, that is to say $f_{i}(z)>0$ requires $z_{3}=x_{3}$ or $z_{3}<x_{3}, z_{1}=0$, and $i \boldsymbol{\epsilon} 1$. 
Next choose $x_{1}^{0} 2\left[1, x_{1}\right]$ and set

$$
D=\delta^{\left(0, x_{2}, x_{3} i 1\right)}-\delta^{\left(0, x_{1}^{0}, x_{3}\right)} .
$$

Now $D$ coincides with $\delta^{\left(0, x_{2}, x_{3} i^{1}\right)}$ on $\left[0, x \mathbf{i} e^{3}\right.$, (31) and Independence of Irrelevant Costs imply that $\varphi\left(D, x \mathbf{i} e^{3}\right)$ is a convex combination of $e^{3}$ and $\frac{1}{2} e^{23}$, hence $\varphi_{1}(D, x \mathbf{i}$ $\left.e^{3}\right)=0$. By (34), $\varphi_{1}(D, x)=0$. This implies that $f_{2}\left(z \mathbf{i} e^{2}\right)=0$ for all $z$ such that $0 \cdot z_{1} \cdot x_{1}, 1 \cdot z_{2} \cdot x_{1}$, and $z_{3}=x_{3}$.

Next pick $x_{2}^{0} 2\left[x_{1}, x_{2}[\right.$ and set

$$
D=\delta^{\left(x_{1}, x_{1}, x_{3}\right)}-\delta^{\left(0, x_{2}^{0}+1, x_{3}\right)} .
$$

As $D$ coincides with $\delta^{\left(x_{1}, x_{1}, x_{3}\right)}$ on $\left[0,\left(x_{1}, x_{2}^{0}, x_{3}\right)\right]$, (31), Independence of Irrelevant Costs and the fact that $f\left(.,\left(x_{1}, x_{2}^{0}, x_{3}\right)\right)$ is contained in $A$ together imply that $\varphi(D$, The cross-subsidizing serial method occupies a central position among the methods described by Lemma 2: see our companion papers Moulin and Sprumont (2002), (2003) for two characterizations of it, based respectively on Distributivity and Coordinality. $\left.\left(x_{1}, x_{2}^{0}, x_{3}\right)\right)=\frac{1}{3} e^{123}$. Now (34) yields $\varphi_{3}(D, x) \cdot \frac{1}{3}$ B y Strong Ranking, this forces $\varphi(D, x)=\frac{1}{3} e^{123}$. Thus $f_{2}\left(z \mathbf{i} \quad e^{2}\right)=0$ for all $z$ such that $0 \cdot z_{1} \cdot x_{1} \mathbf{i} 1$, $x_{1}+1 \cdot z_{2} \cdot x_{2}$, and $z_{3}=x_{3}$. M oreover, $f_{3}\left(z \mathbf{i} e^{3}\right)=0$ for all $z$ such that $z_{1}=0$, $x_{1}+1 \cdot z_{2} \cdot x_{2}$, and $z_{3}=x_{3}$.

$G$ athering our results, we conclude that i) the entire $\ddagger$ ow must go through the edge between $\left(0, x_{1}, x_{3} \mathrm{i} 1\right)$ and $\left(0, x_{1}, x_{3}\right)$, i.e., $f_{3}\left(0, x_{1}, x_{3} \mathrm{i} 1\right)=1$, and ii) within $\mathbf{f} z 2\left[0, x\left[\mathbf{j} z_{3}=x_{3} \mathrm{~g}\right.\right.$, the $\neq$ ow follows the path $\left(0, x_{1}, x_{3}\right) !\left(x_{1}, x_{1}, x_{3}\right) !\left(x_{1}, x_{2}, x_{3}\right)$.

F inally consider

$$
D=\delta^{\left(x_{1} i 1,0, x_{3}\right)}-\delta^{\left(0, x_{1}, x_{3}\right)} .
$$

By the shape of the $\ddagger$ ow $f\left(., x \mathbf{i} \quad e^{1}\right)$ and $(31), \varphi\left(D, x \mathbf{i} \quad e^{1}\right)=\frac{1}{3} e^{123}$. B y the shape of $f(., x)$ and (31), $\varphi(D, x)=e^{3}$, a contradiction to Monotonicity. 